\title{
Knowledge-Based Systems for the Assessment and Management of Bridge Structures: A Review
}

\author{
Ayaho Miyamoto1,2 \\ ${ }^{1}$ Yamaguchi University, Ube, Yamaguchi, Japan \\ ${ }^{2}$ EPFL, Lausanne, Switzerland \\ Email: miya818@yamaguchi-u.ac.jp
}

How to cite this paper: Miyamoto, A. (2021) Knowledge-Based Systems for the Assessment and Management of Bridge Structures: A Review. Journal of Software Engineering and Applications, 14, 505-536. https://doi.org/10.4236/jsea.2021.149030

Received: June 21, 2021

Accepted: September 27, 2021

Published: September 30, 2021

Copyright $\odot 2021$ by author(s) and Scientific Research Publishing Inc. This work is licensed under the Creative Commons Attribution International License (CC BY 4.0).

http://creativecommons.org/licenses/by/4.0/

\section{(c) (i) Open Access}

\begin{abstract}
It is becoming an important social problem to make maintenance and rehabilitation of existing infrastructures such as bridges, buildings, etc. in the world. The kernel of such structure management is to develop a method of safety assessment on items which include remaining life and load carrying capacity. The purpose of this paper is to summarize the finding of up-to-date research articles concerning the application of knowledge-based systems to assessment and management of structures and to illustrate the potential of such systems in the structural engineering. In here, knowledge-based systems include knowledge-based expert systems incorporation with artificial neural networks, fuzzy reasoning and genetic or immune algorithms. Specifically, two modern bridge management systems (BMS's) are presented in the paper. The first is a BMS to assess the performance and derive optimal strategies for inspection and maintenance of concrete bridge structures using reliability based and knowledge-based systems. The second is the concrete bridge rating expert system ( $J$ - $B M S B R E X)$ to evaluate the performance of existing bridges by incorporating with artificial neural networks and fuzzy reasoning.
\end{abstract}

\section{Keywords}

Knowledge-Based System, Infrastructure, Bridge, Maintenance, Management, Expert System, Reliability, Neural Network, Fuzzy Reasoning, Genetic Algorithm

\section{Introduction}

In the field of structure management engineering, a great deal of decision making often depends on the judgment and experience of the domain experts in related fields, such as technologists and engineers. Then an important parameter 
in management for structures is the assessment that should include technological evaluation and economical analyses referring to initial cost and diagnostic technologies for maintenance throughout service life. Since the necessity of developing a computer-aided structure assessment and management system has been pointed out for maintenance, diagnosis, repair and rehabilitation of existing structures [1]. There are multiple processes of damage with a lot of damage factors in existing structures in service. Then in the processes of the structure assessment and management are included a subjective uncertainty of the domain experts such as professional experience, knowledge on structure management, etc. [2].

On the other hand, new technologies are applicable to structure assessment and management such as information technology (IT), artificial intelligence (AI) which based on a soft computing technique, are making remarkable progress. Knowledge-based expert systems (KBESs) with "if-then" rules as one of the artificial intelligence techniques are the most common technology which can be effectively utilized as the structure management supportive tool when such experiences and knowledge are organized, coordinated and developed into a knowledge-based system. As another promising new technology, artificial neural networks (ANNs) originated from a desire to simulate how the human brain processes information. ANNs with the back propagation of error algorithm are able to lean from the past experiences and knowledge by a teacher data. The mechanism of the organization of the neurons and modification of weights of the connections among the neurons in ANNs usually called the machine learning in KBESs (see Figure 1) [3] [4] [5].

The aim of this paper attempts to review and analyze the potential of utilizing knowledge-based expert systems in structure assessment and management and

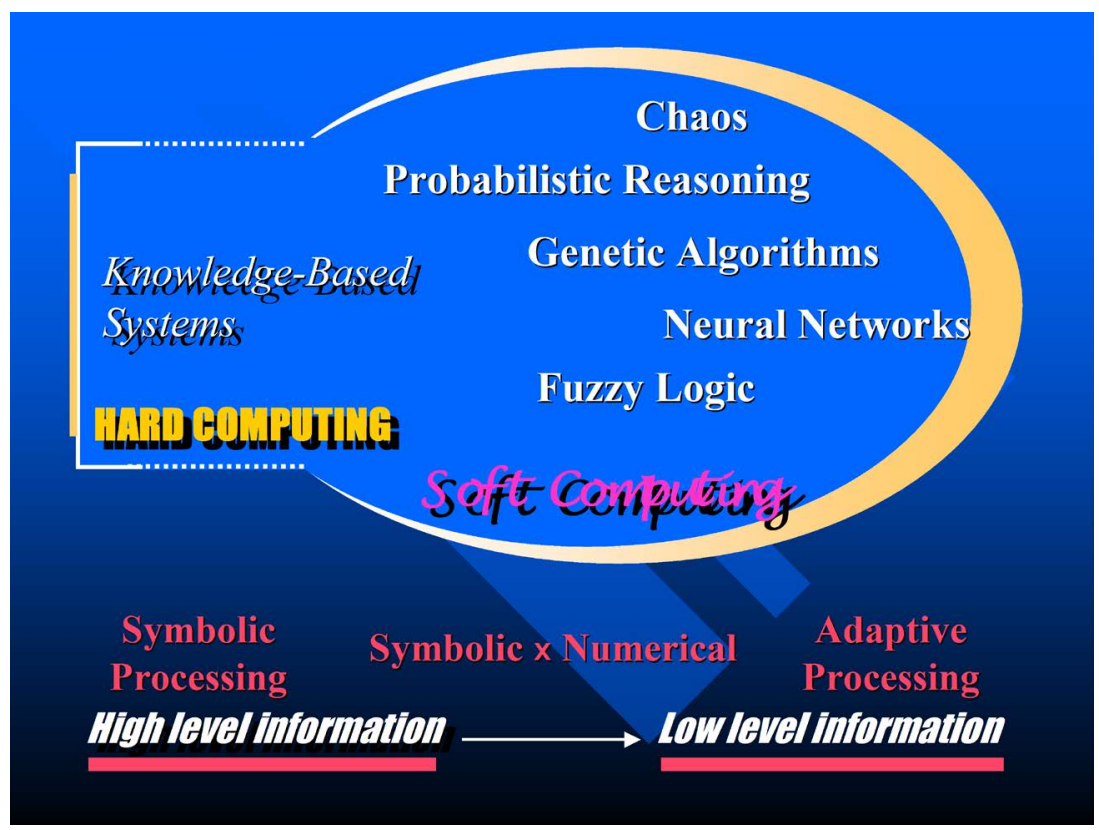

Figure 1. Required technologies for knowledge-based system. 
to make clear the limitations such expert systems based on the qualitative and quantitative information. In here, it reviews the latest applications involving knowledge-based systems for structure assessment and management, including KBESs incorporation with ANNs, fuzzy reasoning and genetic or immune algorithms. Finally, the details of a practical knowledge-based expert system for concrete bridge management are shown as an example of a hybrid system by above-mentioned new technologies. The sections that follow will review two quite different knowledge-based BMSs. The first is a BMS to assess the performance and derive optimal strategies for inspection and maintenance of concrete structures using reliability based [6] and knowledge based systems [7] [8] [9]. The second is the concrete bridge rating expert system (J-BMS BREX) to evaluate the performance of existing bridges by incorporating with artificial neural networks and fuzzy reasoning [10]-[15].

\section{Combined Knowledge-Based and Reliability-Based Systems for Bridge Performance Assessment}

\subsection{Concept of the System}

In this chapter BMS's are discussed with special emphasis on reinforced concrete bridges. However BMS's for prestressed concrete bridges, steel bridges, or composite bridges can be developed in a similar way.

The present bridge management systems are in most cases based on a deterministic approach and the assessment of the reliability or the safety is therefore in general based on subjective statements. In future bridge management systems, we will see a change to stochastically based systems with rational assessment procedures. Future management systems will be computerized and different types of knowledge-based systems will be used. The format of future bridge management systems is illustrated by the EU supported management systems BRIDGE1 and BRIDGE2.

For many years it has been accepted that steel bridges must be maintained due to the risk of corrosion of steel girders etc. The situation is a little different for reinforced concrete bridges. Although a vast majority of reinforced concrete bridges have performed satisfactorily during their service life, numerous instances of distress and deterioration have been observed in such structures in recent years. The causes of deterioration of reinforced concrete bridges are often related to durability problems of the composite material. One of the most important deterioration processes, which may occur in reinforced concrete bridges, is reinforcement corrosion, caused by chlorides present in de-icing salts and/or carbonation of the concrete cover zone.

Improved stochastic modelling of the deterioration is needed to be able to formulate optimal strategies for inspection and maintenance. However, such strategies will only be useful if they are combined with expert knowledge. It is believed that future management systems will be expert systems or at least knowledge-based systems [7] [8] [9]. 


\subsection{Optimal Strategies for Inspection and Maintenance of Bridges}

Diagnostic methods: Diagnosis of bridges showing signs of functional or structural deterioration is the first step that has to be taken before making any decisions regarding maintenance or repair. It is necessary to define clearly what are the damage problems. It is very costly to start diagnosis without knowing which information one wants to gather. When the diagnostic method (or methods) is selected, it is necessary to gather the know-how, equipment, manpower and facilities needed. Diagnostic work is usually disruptive for the normal functioning of the bridge and must be limited as much as possible in time and space.

Correlations between defects and diagnostic methods. A correlation matrix between the diagnosis methods and the defects can be established so that each line represents a defect and each column a diagnostic method. At the intersection of each line and column a number representing the correlation between defect and diagnostic method can then be introduced. Such a matrix helps the inspector in choosing the best inspection method, as a function of the detected defect.

\subsection{Development of Optimal Strategies}

Inspection strategies: Methods and computer programs for determining rational inspection and maintenance strategies for bridges are developed. The optimal decision is based on the expected benefits and the total cost of inspection, repair, maintenance and complete or partial failure of the bridge. Furthermore, the reliability has to be acceptable during the expected lifetime. Inspections of bridges are in this BMS divided into three types:

- Current inspections, which are performed at a fixed time interval, e.g. 15 months. The inspection is mainly a visual inspection.

- Detailed inspections are also done at a fixed time interval. The time interval is a multiple of the current inspection time interval, e.g. 5 years. The detailed inspections are visual inspections but can also include non-destructive in-situ tests.

- Structural assessments are only performed when a current or detailed inspection shows some serious defects, which require a more detailed investigation. The structural assessment can include laboratorial tests, in-situ tests with non-portable equipment, static and dynamic load tests. The tests are usually very costly compared with the other two inspection types.

Maintenance and repair decision systems. It is convenient to divide that part of the decision system, which is used to assist in maintenance and repair planning into two subsystems. The maintenance subsystem deals with maintenance repair techniques and small repair, i.e. repair of unimportant structural defects. Generally, this subsystem is always used after a current or detailed inspection. The repair subsystem helps choosing the best option of structural repair when an important deficiency that impairs the functionality of the bridge is detected. It is basically an economic decision (based obviously on structural and traffic engineering data) in which the costs are quantified. Generally, this sub- 
system is used after a structural assessment.

\subsection{Application of Expert Knowledge}

The objective of using knowledge-based system technology in bridge management is to produce a software tool to assist bridge inspectors as well as engineering experts in their tasks of assessing and improving the reliability of concrete bridges.

Architecture: The first step is to identify the various software subsystems and the relations between them i.e. the software architecture that will set the basis for the development of the knowledge-based systems. It is natural in bridge management to develop two different modules aimed at different goals. The first provides technical support to the inspector during the inspection process at the bridge site. The second assists the engineer in the analysis of the safety of bridges as well as in the selection of maintenance and repair methods.

Software modules: A number of software modules interact with the knowledge-based systems through specifically designed data files:

- Updating analysis: Based on inspection information and other new information the reliability estimates and the data in the databases is updated.

- Reliability analysis: The reliability of the bridge is evaluated as a function of time.

- Structural analysis: The system is open so that the user is able to use any finite element software.

- Inspection program: Based on the data in the databases and the reliability estimates the optimal time for the next inspection is calculated using the updating module.

The next step is to identify the representation schemes and inference mechanisms best suited for the implementation of the knowledge-based systems.

Implementation of the knowledge based system: As mentioned earlier in bridge management it is convenient to have at least two systems, namely one to be used in the inspection phase and one to be used during maintenance and for repair decisions. The first system is highly based on "correlation matrices". Correlation matrices are defined for: defects/diagnostic methods, defects/causes and defects/repair methods. A pseudo-quantitative classification of the type no correlation, low and high correlation is used. Correlation between defects as well as diagnostic and repair methods is also needed. Each matrix is e.g. be organized so that each line represents a defect and each column a possible diagnosis method, cause or repair method. At the intersection of each line and column a number representing the correlation between defect and possible element of reference is introduced.

It is important for the applicability of the knowledge-based system that it gives all the information needed during and after inspections. Such information are: general information about the bridge, related diagnostic methods, probable causes, associated defects and provisional defect report. 
Databases: A crucial task in the development of knowledge-based systems is the definition of the databases. An exhaustive study of the data collected for concrete bridges, both at the design stage and after it has been constructed must be provided. At relevant moments of the bridge's service life (usually after construction and after important rehabilitation work is performed), its real situation must be thoroughly described so that future inspections have something to relate to. When the database definition is completed then the set of parameters required for the reliability estimation, the cost optimization, and additional bridge parameters dealing with the bridge repair cost and corrosion descriptive parameters are added.

Most existing bridge management databases are insufficient for e.g. reliability assessment and for implementing modern decision-making tools.

Expert modules: A number of expert modules are needed to define the architecture of the knowledge-based system: database module, inspection module and a decision module. The decision is divided into a number of sub-modules such as: a maintenance/small repair sub module, an inspection strategy sub module and a repair/upgrading/replacement sub module.

The inspector's functionalities: The inspector must be able to perform activities like:

- Review all the information contained in the database of the bridges. Different types of data are recorded for each bridge: identification and bridge site information, design information, budget information, traffic information, strength information, load information, deterioration information, factors that model the costs and data for the cross-sections defined for the bridge.

- Define new cross-sections.

- Receive technical support regarding the most appropriate diagnostic methods to be used in order to conclude about the existence of a defect.

- Receive technical support regarding the possible causes responsible for a defect.

- Record the results of the inspection.

The inspection engineer's functionalities. The inspection engineer must at his office be able to:

- View the inspection results recorded at any previous inspection performed in any of the bridges of the database.

- Enter the data of a bridge in the bridge's database.

- View the data of a bridge and edit it.

- Define new critical cross-sections for any of the bridges in the database.

- Complete the data of the defects detected at the inspection by describing the defect in greater detail and by entering the results of the tests performed.

- Update data for the cross-sections and inspection results after repair.

\subsection{Bridge Management Systems for Concrete}

In this section some important issues related to advanced BMSs are discussed 
namely:

- Deterioration of bridges

- Stochastic modeling of failure modes

- Stochastic modeling of the inspection

- Stochastic modeling of repair

- Updating techniques

- Reliability analysis.

Deterioration of bridges: Corrosion is one of the most important deterioration mechanisms for steel as well as reinforced concrete bridges. In this section a stochastic model for corrosion of reinforcement in reinforced concrete bridges is shown.

Fick's law of diffusion often models the rate of chloride penetration into concrete

$$
\frac{\partial C(x, t)}{\partial t}=D_{C} \frac{\partial^{2} C(x, t)}{\partial x^{2}}
$$

where, $D_{C}$ is the chloride diffusion coefficient, $x$ is the distance from the surface and $t$ is time. The solution of Equation (1) is

$$
C(x, t)=C_{0}\left\{1-\operatorname{erf}\left(\frac{x}{2 \sqrt{D_{C} \cdot t}}\right)\right\}
$$

where, $C(x, t)$ is the chloride content at the distance $x$ from the surface and at the time $t, C_{0}$ is the initial chloride content, and $\operatorname{erf}()$ is the error function. The corrosion initiation period

$$
T_{I}=\frac{\left(d_{1}-D_{1} / 2\right)^{2}}{4 D_{C}}\left(\operatorname{erf}^{-1}\left(\frac{C_{c r}-C_{0}}{C_{i}-C_{0}}\right)\right)^{-2}
$$

where, $C_{i}$ is the initial chloride concentration, $C_{c r}$ is the critical chloride concentration, and $d_{1}-D_{1} / 2$ is the concrete cover. The diameter $D_{1}(t)$ of the reinforcement bars at the time $t$ after initiation of corrosion can be modeled by

$$
D_{1}(t)=D_{1}(0)-C_{\text {corr }} i_{\text {corr }}(t)
$$

where, $D_{1}(0)$ is the initial diameter, $C_{\text {corr }}$ is a corrosion coefficient, and $i_{\text {corr }}$ is the rate of corrosion.

Stochastic modeling of failure modes: A number of failure modes for structural elements must be modeled. In this section is shown as illustration modeling of an ultimate limit state (ULS) and a serviceability limit state for a concrete slab bridge namely [8]:

- An ultimate limit state (ULS): collapse limit state (using yield line analysis).

- A serviceability limit state (SLS): crack width limit state (using linear elastic analysis).

The following safety margin can be used for the collapse limit state:

$$
Z_{c}=V_{c} E_{D}-W_{D}
$$

where, $V_{C}$ is a model uncertainty variable, $E_{D}$ is the energy dissipated in yield 
lines, and $W_{D}$ is the work done by the applied loads.

The basic variables used in the yield line ULS are: thickness of slab, cube strength of concrete, density of concrete, depth of reinforcement, yield strength of reinforcement, and two load parameters.

Cracking shall be limited to a level that will not impair the proper functioning of the structure or cause its appearance to be unacceptable. In the absence of specific requirements (e.g. water tightness), it may be assumed that limitation of the maximum design crack width $W_{\max }$ to about $0.3 \mathrm{~mm}$ will generally be satisfactory for reinforced concrete members with respect to appearance and durability.

A crack width limit state can be formulated by

$$
Z_{w}=W_{\max }-Z_{w} W_{d}
$$

where, $W_{\max }$ is the maximum allowable crack, $W_{d}$ is the design crack width, and $Z_{d}$ is a model uncertainty stochastic variable.

Stochastic modeling of the inspection: Two types of uncertainty in the models for inspections must be considered. The first type of uncertainty is related to the uncertainty (reliability) of an inspection method, i.e., how good is an inspection technique to detect a defect if a defect is present and what is the risk that the inspection method indicates a defect when there is no defect (false alarm). The second type of uncertainty is related to the measurement uncertainty when a detected defect is being quantified. Stochastic models must be derived for the most important inspection methods.

Stochastic modeling of repair. Repair implies that new and/or modified values of parameters are needed to model the behavior of the bridge after the repair. In relation to stochastic modeling of repair the quantities can be divided into the following groups:

- Quantities (deterministic or stochastic), which are the same before and after repair.

- Quantities, which can be modeled by deterministic variables. The values for these quantities are known rather precisely after the repair.

- Quantities, which can be considered new outcomes of the old stochastic variables used before the repair. A variable of this type is modeled by introducing a new stochastic variable with the same distribution function but statistically independent of the old stochastic variable.

- Quantities modeled by new stochastic variables correlated or not correlated with the old stochastic variables.

In addition to the above models it can be relevant to update the distribution functions of the stochastic variables when observations are obtained in connection with the repair. The following important structural repair types must be modelled: concrete patching (with deteriorated concrete removal), concrete patching (with reinforcement cleaning), concrete patching (with reinforcement splicing/replacement) and concrete encasing (with reinforcement splicing/ replacement). 
Updating techniques: When new information becomes available the estimates of the probability of failure (and the reliability) of structures can be updated. New information can be divided in three types:

- Sample information on basic variables.

- General information on stochastic variables.

- Linguistic information.

When new information is available as samples of one or more stochastic basic variables Bayesian statistical methods are used to obtain updated (predictive) distribution functions of the stochastic variables.

In some cases the information obtained by measurements is not directly related to a basic stochastic variable. The information is generally modeled by using a stochastic variable, which is a function of the basic stochastic variables. The event margin is a stochastic variable and it is therefore possible to estimate the probability that the event occurs. Further, this type of information can be used to update the probability of failure of a structural element.

Basic variable updating is performed within the framework of Bayesian statistical theory [8]. The updating based on general information is mainly based on the Bayesian methods suggested by Refs. [16] [17].

Let the density function of a stochastic variable $X$ be given by $f_{X}(x, \Theta)$, where, $\Theta$ are parameters defining the distribution of $X$. The parameters $\Theta$ are treated as uncertain parameters (stochastic variables). $f_{X}(x, \Theta)$ is therefore a conditional density function $f_{X}(x \mid \Theta)$. The initial (or prior) density function for $\Theta$ is called $g_{\Theta}^{\prime}(\theta)$.

When an inspection is performed $n$ realizations $\bar{X}^{*}=\left(x_{1}, \cdots, x_{n}\right)$ of the stochastic variable $X$ are obtained. The inspection results are assumed to be independent. An updated density function $\Theta$ taking into account the inspection results is then defined by

$$
g_{\Theta}^{\prime \prime}\left(\theta \mid \bar{x}^{*}\right)=\frac{f\left(\bar{x}^{*} \mid \theta\right) g_{\Theta}^{\prime}(\theta)}{\int f_{n}\left(\bar{x}^{*} \mid \theta\right) g_{\Theta}^{\prime}(\theta) \mathrm{d} \theta}
$$

where, $f_{X}\left(x \mid \bar{x}^{*}\right)=\prod_{i=1}^{n} f_{X}\left(x_{i} \mid \theta\right)$.

The updated density function of $X$ taking into account the realizations $\bar{x}^{*}$ is then obtained by

$$
f_{X}\left(x \mid \bar{x}^{*}\right)=\int f_{X}(x \mid \theta) g_{\Theta}^{\prime \prime}\left(\theta \mid \bar{x}^{*}\right) \mathrm{d}(\theta)
$$

In the knowledge-based systems the functions $g_{\Theta}^{\prime}(\theta), g_{\Theta}^{\prime \prime}(\theta)$ and $f_{X}\left(x \mid \bar{x}^{*}\right)$ are implemented for several distributions.

Reliability analysis: The reliability of the bridge is measured using the reliability index $\beta$ for a single failure element or for the structural system (the bridge) [18] [19]. The reliability is assumed to decrease with time due to the deterioration. The failure modes can e.g. be stability failure of columns, yielding or shear failure in a number of critical cross-sections of the bridge. If a system modeling is used then it is assumed that the structure fails if any one of these failure modes fails, i.e. a series system modeling is used. 
It is assumed that uncertain quantities like loading, strength and inspection results can be modeled by $N$ stochastic variables $\bar{X}=\left(X_{1}, \cdots, X_{N}\right)$. The structure is modeled by $m$ potential failure modes $F_{i}, i=1,2, \cdots, m$. Failure mode $i$ is described by a safety margin.

$$
M_{F_{i}}=M_{F_{i}}(\bar{X}, t)
$$

The element reliability index, $\beta_{i}(t)$ at the time $t$ for failure mode $F_{i}$ is connected to the probability of failure $P_{F_{i}}(t)$ by Ref. [18].

$$
\beta_{i}(t)=-\Phi^{-1}\left(P_{F_{i}}(t)\right)
$$

where, $\Phi$ is the standard normal distribution function. The probability of failure $P_{F_{i}}(t)$ in the time interval $[0, t]$ is determined from

$$
P_{F_{i}}=P\left(M_{F_{i}} \leq 0\right)
$$

In Ref. [8] is in an example shown how a reliability assessment of a concrete bridge taking into consideration corrosion of the reinforcement may be performed. The example is based on an existing UK bridge, but some limitations and simplifications are made.

A plastic collapse analysis and estimation of the load are performed using the COBRAS program [20]. The reliability analysis (element and system) is done using the programs "RELIAB01" and "RELIAB02" [21] [22]. The "RELIAB" and "COBRAS" programs have been interfaced and include an optimization algorithm to determine the optimal yield line pattern for each iteration of the reliability analysis. The estimation of the deterioration of the steel reinforcement is based on the program "CORROSION" [23].

\subsection{Bridge1 \& Bridge2 BMS}

Introduction: Some results from the research project "Assessment of Performance and Optimal Strategies for Inspection and Maintenance of Concrete Structures using Reliability Based Expert Systems", supported by EU within the BRITE/EURAM research program, is presented in this chapter.

The main objective of the project was to optimize strategies for inspection, maintenance and repair of reinforced concrete bridges by developing improved methods for modelling the deterioration of existing as well as future structures using reliability based methods and knowledge based systems.

Reliability assessment. In this bridge management system the probability of failure is estimated using the reliability program RELIAB.

The system reliability index $\beta^{s}(t)$ is connected to the probability of failure $P_{F_{i}}(t)$ of the series system in the time interval $[0, t]$ by

$$
\beta^{s}(t)=-\Phi^{-1}\left(P_{F}(t)\right)
$$

where, the probability of failure, $P_{F}(t)$ is determined by the approximation [19].

$$
P_{F}(t) \approx 1-\Phi_{m}(\bar{\beta}(t), \overline{\bar{\rho}}(t))
$$


where, $\bar{\beta}=\left(\beta_{1}, \cdots, \beta_{m}\right)$ and $\overline{\bar{\rho}}(t)$ are a matrix whose elements are the correlation coefficients between the linearized failure margins of the elements in the series system. $\Phi_{m}$ is the m-dimensional normal distribution function.

In the bridge management systems BRIDGE1 and BRIDGE2 the updating of stochastic variables etc. is performed using the techniques described earlier.

Functionalities of BRIDGE1 and BRIDGE2: The expert system is divided into two modules BRIDGE1 and BRIDGE2 which are used in two different situations, namely by the inspector of the bridge during the inspection at the site and after the inspector has returned to his office.

During the inspection the knowledge based system will supply information on: the causes of observed defects, appropriate diagnostic methods, and related defects. Further, the inspector will be asked to record the inspection results so that they can be used later for e.g. assessment of the reliability of the bridge and in the decision whether a detailed structural assessment is needed.

A detailed analysis of the state of the bridge after an inspection is performed when the inspector has returned to his office, and after testing in the laboratory has been performed. The output of the analysis includes an updated estimation of the reliability of the bridge, decision whether a structural assessment should be made, decision whether to repair or not, relevant repair procedures, and the time for repair. Expert knowledge is used to improve the quality of the decisions.

Application of BRIDGE1 and BRIDGE2: The general inspection, maintenance and repair model from inspection no. $i$ at time $t_{i}$ to inspection no. $i+$ 1 at the time $t_{i+1}=t_{i}+\Delta t$ is indicated in Figure 2, where also the application of the modules BRIDGE1 and BRIDGE2 is shown.

The symbols used in Figure 2 are:

C: Current inspections are performed at a fixed time interval, e.g. 15 months.

D: Detailed inspections are also periodic at a fixed time interval, which is a multiple of the current inspection time intervals, e.g. 5 years.

A: Structural assessments are only performed when a current or detailed inspection shows some serious defects, which require a more detailed investigation.

M: Maintenance and repair of minor defects.

R: Structural repair.

B1: Application of BRIDGE1 during the inspections.

B2(M): The maintenance subsystem in BRIDGE2 assists in the selection of maintenance work and repair of minor structural defects to be performed.

B2(I): The inspection module in BRIDGE2 assists in selecting the next type of inspection.

$\mathrm{B} 2(\mathrm{R}):$ The repair subsystem in BRIDGE2 assists in selecting the best repair technique. The selection is based on economic considerations and expert knowledge.

After a current or a detailed inspection BRIDGE2 is used to rate the maintenance and minor repair work needed and to decide if a structural assessment has 


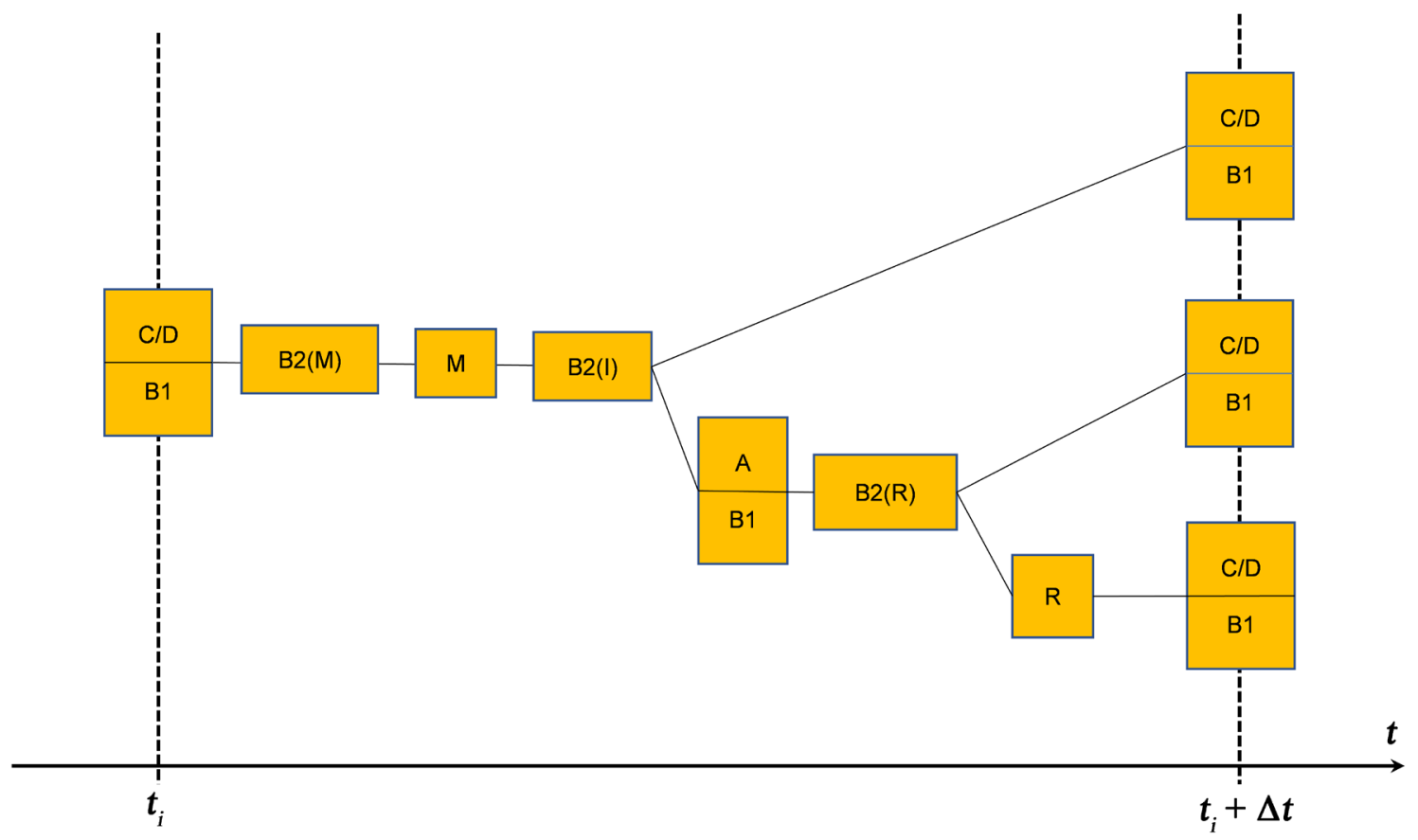

Figure 2. Inspection, maintenance and repair models.

to be performed. The decision is based partly on estimates of the reliability of the bridge and partly on expert knowledge. The decision does not include economic considerations.

After a structural assessment BRIDGE2 is used to decide if a repair has to be performed and also to give the optimal point of time for the repair. Expert knowledge as well as numerical algorithms is used. The decisions are partly based on a cost-based optimization where different repair possibilities (selected by expert knowledge) and no repair are compared.

Decision model with regard to structural assessment. A structural assessment is recommended if the updated reliability index for the bridge $\beta^{U}$ is smaller than or equal to a minimum reliability index $\beta^{\text {min }}$ as shown in Figure 3. If the updated reliability index for the bridge is greater than the minimum reliability index then the decision is taken based on expert knowledge.

Let $t_{i}$ be the time of a periodic inspection and let the updated reliability index at the time $t$ be $\beta\left(t, t_{i}\right)$. The general decision model with regard to the structural assessment can then be formulated as:

- If $\beta\left(t_{i+1}, t_{i}\right)>\beta^{\text {min }}$ then the inspection at the time $t_{i+1}$ should be a current or detailed inspection unless the damage is so serious that a structural assessment is needed. This decision is based on expert knowledge. $\beta^{\min }$ is the minimum acceptable reliability index (e.g. 3.72).

- If $\beta\left(t_{i+1}, t_{i}\right) \leq \beta^{\min }$ then a structural assessment should be performed before the next periodic inspection.

Modeling of repair. After a structural assessment it must be decided whether the bridge should be repaired and if so, how the repair is to be performed. Solution of this problem requires that all future inspections and repairs be taken into account. 


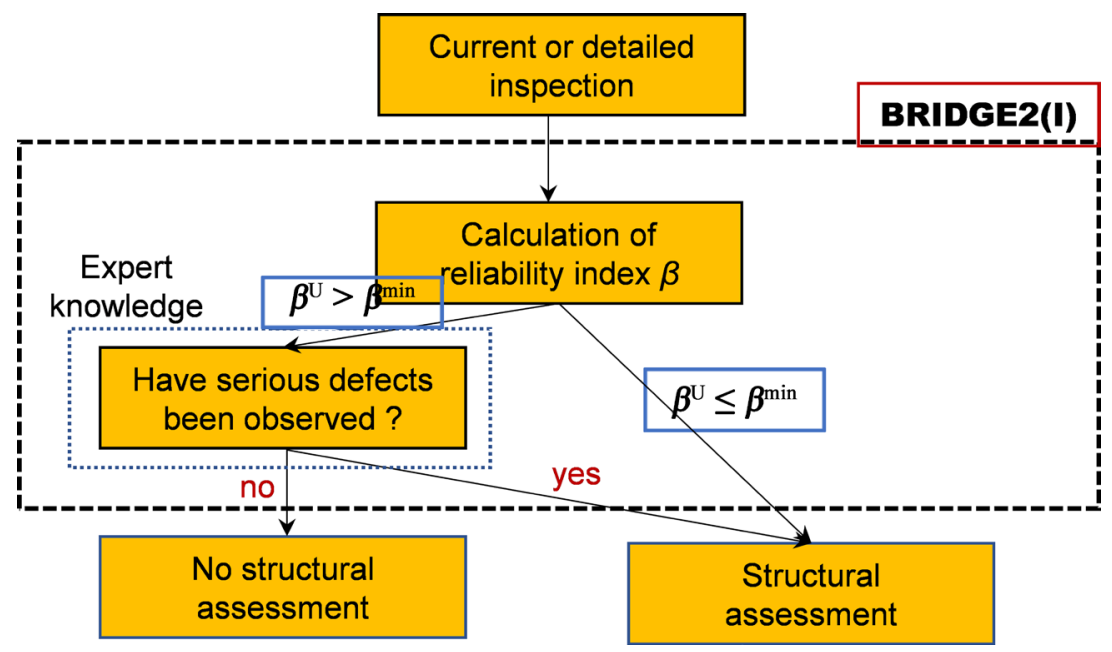

Figure 3. Decision model for structural assessment.

In order to decide which repair type is optimal after a structural assessment; the following optimization problem is considered for each repair technique:

$$
\begin{gathered}
\max _{T_{R}, N_{R}} W\left(T_{R}, N_{R}\right)=B\left(T_{R}, N_{R}\right)-C_{R}\left(T_{R}, N_{R}\right)-C_{F}\left(T_{R}, N_{R}\right) \\
\text { s.t. } \beta^{U}\left(T_{L}, T_{R}, N_{R}\right) \geq \beta^{\min }
\end{gathered}
$$

where the optimization variables are the expected number of repair $N_{R}$ in the remaining lifetime and the time $T_{R}$ of the first repair. $W$ is the total expected benefits minus costs in the remaining lifetime of the bridge. $B$ is the benefit. $C_{R}$ is the repair cost capitalized to the time $t=0$ in the remaining lifetime of the bridge. $C_{F}$ is the expected failure costs capitalized to the time $t=0$ in the remaining lifetime of the bridge. $T_{L}$ is the expected lifetime of the bridge. $\beta^{U}$ is the updated reliability index. $\beta^{\min }$ is the minimum reliability index for the bridge (related to a critical element or to the total system).

The repair decision is then based on the results of solving this optimization problem but also on expert knowledge.

Elicitation. Expert knowledge plays a very important role in this project.As a simple example decisions resulting in Structural assessment or No structural assessment are described for one of the most important defects namely "rust stain".

If rust stain is observed at the inspection then the following question is asked:

Question 1: What is the extent of rust stain?

\section{Possible answers to question 1:}

1) Single rust stains $\Rightarrow$ No structural assessment (it is assumed that single rust stains do not question the structural safety or the global functionality of the bridge).

2) Locally many rust stains $\Rightarrow$ question 2 .

3) Widespread rust stains $\Rightarrow$ Structural assessment (it is assumed that there is a global corrosion of the reinforcement in the bridge).

If item 2 is the result of question 1 then question 2 is asked. 
Question 2: What is the location of rust stains?

\section{Possible answers to question 2:}

1) A critical place regarding humidity $\Rightarrow$ Structural assessment (a place is critical if it e.g. is exposed to splash of water from cars passing under the bridge).

2) Near places where maximum moments occur $\Rightarrow$ Structural assessment.

3) Other places $\Rightarrow$ No structural assessment.

The result of the elicitation is illustrated in Figure 4.

BRIDGE1: As mentioned earlier, the expert system module BRIDGE1 is used at the bridge site during an inspection. This expert system module contains useful information concerning the bridge inspected and the defects observed. The information includes: general information about the bridge, appropriate diagnostic methods for each defect, probable causes for each defect, and other defects related to a defect. It is also possible to create a provisional defect report.

The general information about the bridge stored in the database for the selected bridge can be reviewed. The database contains information about: bridge site, design, budget, traffic, strength, load, deterioration, factors that model the costs, and the cross-sections entered for the bridge.

New cross-sections can be entered for the selected bridge. The information stored in the database for each cross-section contains: cross-section identification, geometry of cross-section (detailed description of the reinforcement layers for cross-sections in the deck), failure mode, and load data. Technical support can be provided for a defect.

The technical support includes a list of diagnostic methods that can be used to observe a selected defect. The list is divided into high and low correlated diagnostic methods for the selected defect.

A list of defects associated with the selected defect is also included. This list is very useful since the defects, which can be found with a high probability, can be reviewed if the selected defect is observed. Measures for the correlations between the selected defect and the related defects are shown.

BRIDGE2: The expert system module BRIDGE2 is used to make a detailed analysis of the bridge after an inspection when testing has been performed in the

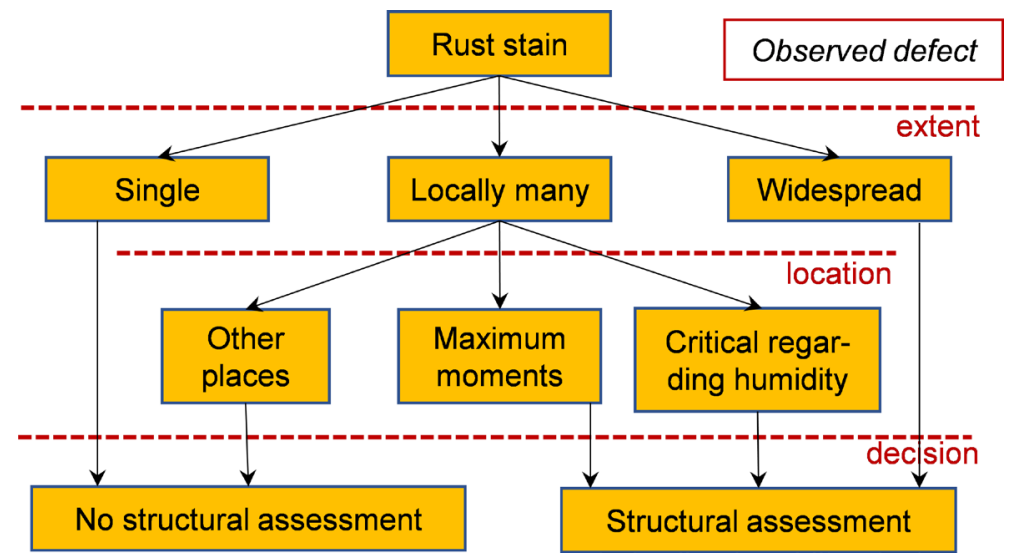

Figure 4. Elicitation. 
laboratory. New bridges and cross-sections can be entered into the database and existing bridges and cross-sections can be edited. For the bridges in the database the following options are available: review provisional defect reports, enter inspection results, estimate the reliability index, plan maintenance work and estimate costs, plan structural repair work and estimate costs, and review the agenda of inspection for one bridge or all bridges. Further, the database can be updated after repair.

New bridges can be entered and existing bridges can be edited. The general information about the bridges stored in the database contains information about: bridge site, design, budget, traffic, strength, load, deterioration, factors that model the costs, and the cross-sections entered for each bridge.

After an inspection the provisional defect reports recorded at previous inspections can be reviewed. A description of the detected defects and measurements of diagnostic methods can be entered. After a repair the databases can be updated.

The integrated FORTRAN program RELIAB can estimate the reliability index for the bridge. The reliability index when no inspection results are taken into account and the updated reliability index when all inspections performed for the bridge are both taken into account can be estimated.

The following sub modules are integrated into BRIDGE2:

- $B R I D G E 2(M)$ is the maintenance/small repair sub module. This sub module assists in selecting the maintenance work and repair of minor structural defects to be performed and estimates the maintenance costs. The defects are rated based on the defect classification in terms of rehabilitation urgency, importance of the structure's stability, and affected traffic recorded during the inspection.

- BRIDGE2(I) is the inspection strategy sub module. This assists in the decision whether a structural assessment is needed before the next periodic inspection. The decision made in BRIDGE2(I) is mainly based on the updated reliability index for the bridge calculated by RELIAB. If the value of the updated reliability index for the bridge is acceptable then each of the defects detected at the latest periodic inspection and the combination of defects are investigated based on a cost-benefit analysis by the FORTRAN program INSPEC.

The FORTRAN program RELIAB can be used to estimate the reliability of a bridge. The FORTRAN program INSPEC can be used to estimate the optimal repair time and number of repairs for a given repair method. The estimation is based on a cost-benefit analysis for the bridge. The total expected benefits minus expected repair and failure costs in the remaining lifetime of the bridge is optimized.

\section{Practical Hybrid Knowledge-Based Expert Systems for Concrete Bridge Management}

\subsection{Concept of the System}

The author has been developing a knowledge-based expert system which can be 
used to evaluate the performance of existing concrete bridges on the basis of knowledge and experience acquired from domain experts [10]-[15] [24] [25]. The proposed expert system is called the Concrete Bridge Rating EXpert System ( $J$-BMS BREX). The objective of the system is to evaluate the present performance of target bridge members in terms of factors such as serviceability, load-carrying capability, and durability. The input data for rating a concrete bridge are the technical specifications of the target bridge, environmental conditions, traffic volume, and other subjective information that can be obtained through simple visual inspection. In this study, load-carrying capability and durability are used to estimate serviceability. Load-carrying capability is defined as the aspect of bridge performance that is based on the load-carrying capacity of a bridge member, and durability is defined as the ability of a bridge member to resist material deterioration and is based on the rate of material deterioration of the member. These two aspects of bridge performance are applied as indices for considering the necessity of performing maintenance on deteriorated bridges. Specifically, load-carrying capability is applied as an index for estimating the necessity of strengthening, and durability is applied as an index for estimating the necessity of repair.

In the expert system, the performance of a target bridge is evaluated according to a diagnostic process, which is modeled on the inference process domain experts employ for rating an existing concrete bridge. This process is expressed by a hierarchical structure and has twelve main judgment items. The ultimate goal of this process is "serviceability." The hierarchical structure expresses the relationship between judgment items and input data, such as inspection data and technical specification data, or between judgment items. In practice, these relationships are expressed by "If-then" rules with fuzzy variables. Consequently, the fuzzy inference of the expert system is drawn from these rules. Naturally, these rules could be written directly into a computer in a computer language. In this study, however, these rules are implemented in a computer after a set of the rules relating judgment items and input data or relating judgment items is transformed to a hierarchical neural network. In other words, hierarchical neural networks identify a diagnostic process. The system can easily refine the knowledge base; that is, "If-then" rules with fuzzy variables, by use of a machine learning method. More specifically, the system refines the knowledge base by applying the "Back-Propagation" method [4]. Therefore, since the network is capable of performing fuzzy inference and machine learning, the system can be called a Neuro-Fuzzy expert system. Generally, although a neural network is a powerful machine learning tool, the inference process of a neural network becomes a "black box," which renders the representation of knowledge in the form of rules impossible. However, the hierarchical neural network proposed in the present study contributes to prevent an inference process from becoming a black box. As described later, the effectiveness of the hierarchical neural network and machine learning method was verified by comparison of the diagnostic results of bridge experts and those of the proposed system. 


\subsection{Knowledge-Based Expert System for Existing Concrete Bridges}

In the expert system, the target bridge is diagnosed according to a diagnostic process, which is modeled on the inference mechanism used by domain experts for rating bridges (see Figure 5). In a previous study, the authors used the Fuzzy Structural Modeling (FSM) method [5] to create the diagnostic process for main girders and slabs. Each process employs twelve main judgment items. These judgment items are evaluated by about 90 input data items, such as technical specifications, traffic volume, and results of visual inspection. The process is a hierarchical structure in which the ultimate goal is "serviceability."

For instance, Figure 5 shows the diagnostic process for main girders. The lowest-rated judgment items, such as "Condition state of cracking" and "Condition state other than cracking," are first evaluated by use of input data such as visual inspection data and technical specifications. The "Condition state of cracking" is evaluated from inspection data such as [Crack conditions] and [Maximum crack width $(\mathrm{mm})$ ]. Next, the higher-rated judgment items, such as "flexural cracks," "shear cracks," and "material deterioration," are diagnosed from the results of lower judgment items and/or input data. The damage degree of "flexural cracks" is determined from the results of "Condition state of cracking"

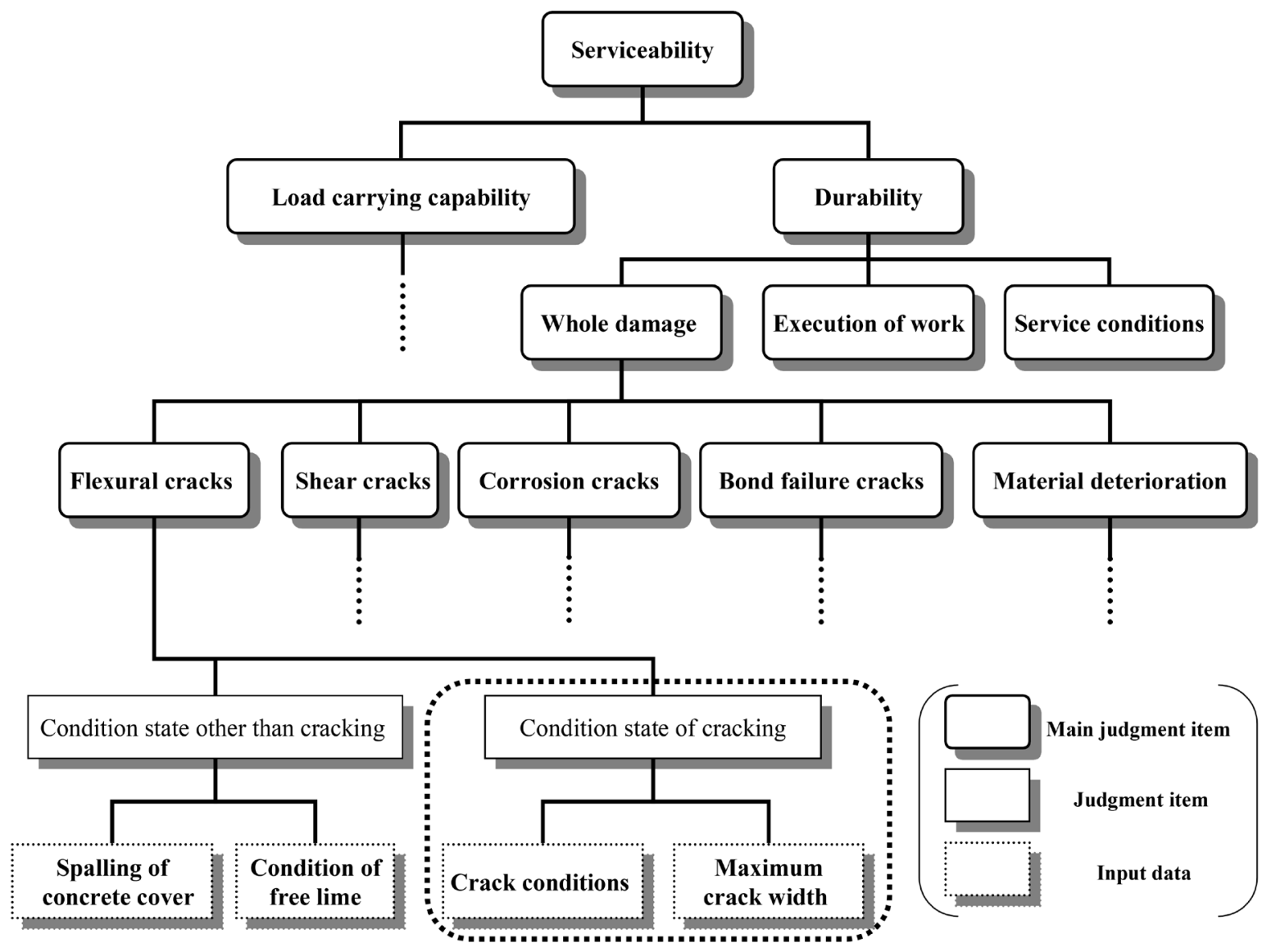

Figure 5. Example of the diagnostic process for main girder. 
and "Condition state other than cracking." Then, the higher-rated judgment items, such as "whole damage," "execution of work," and "service conditions," are also evaluated from the results of lower judgment items and/or input data. The final judgment item is "serviceability," which is evaluated according to the results of "load-carrying capability" and "durability." Each of these judgment items is assigned a soundness score, on a scale of 0 - 100, which is output from the expert system. The output score is categorized into one of five groups: 0 $12.5,12.6$ - 37.5, 37.6 - 62.5, 62.6 - 87.5 and 87.6 - 100. These groups are classified as "dangerous," "slightly dangerous," "moderate," "fairly safe," and "safe," respectively. In the present study, "safe" indicates that the bridge has no problems; "fairly safe" indicates no serious damage; "moderate" indicates the presence of some damage that requires continuous inspection; "slightly dangerous" indicates that the bridge should be repaired and/or strengthened; and "dangerous" indicates that the bridge should be removed from service and requires rebuilding. In the expert system, the relationships between judgment items and input data and those between judgment items are expressed by "If-then" rules with fuzzy variables. In addition, by introduction of machine learning into the expert system, these rules are implemented by hierarchical neural networks. A hierarchical network expresses a set of rules for evaluating a judgment item.

\subsection{Knowledge Representation}

The expert system evaluates the performance of a target bridge according to the diagnostic process, which expresses the relationships between judgment items and input data or between judgment items, as shown in Figure 5. In the knowledge base of the system, the diagnostic process is stored in the form of "If-then" rules with fuzzy variables. Consequently, these rules enable the system to perform fuzzy inference. The knowledge representation of the system is as follows:

$$
R^{i} \text { : if } x_{1} \text { is } A_{1} \text { and } \cdots \text { and } x_{m} \text { is } A_{m} \text { then } y \text { is } B_{i}
$$

where, $R^{i}: i$ th fuzzy rule.

$x_{1}, \cdots, x_{m}$ : input items (input data such as technical specifications and results of visual inspection).

y. output item (diagnosis item; that is, judgment item).

$A_{1}, \cdots, A_{m}$ : fuzzy variables.

$B_{i}$ : constant (soundness score on the scale of 0 - 100).

For example, If ([Crack condition] is serious) and ([Maximum crack width] is huge) then ([Condition state of cracking] is 0.0 ). This rule is used in order to evaluate the judgment item "Condition state of cracking."

Fuzzy inference process. This section describes in detail the fuzzy inference process performed in the expert system. The portion of Figure 5 enclosed in a dotted box; namely, the inference process that evaluates "Condition state of cracking," is explained as an instance. Table 1 shows the fuzzy rules for evaluating the judgment item" Condition state of cracking." For example, Rule No. 12 expresses the following fuzzy rule; If ([Crack conditions] is OK) and ([Maxi- 
mum crack width] is OK) then ([Condition state of cracking] is 100.0). Since these rules employ some fuzzy expressions; namely, antecedents of the rules employ some fuzzy propositions, the initial form of membership functions for fuzzy rules must be prepared. Figure 6 shows an example of the assigned membership functions related to the fuzzy rules for evaluating "Condition state of cracking." Table 2 shows an excerpt of the inspection sheet used for the system. The solid circles indicate inspection results. The inference process of "Condition state of cracking" diagnosis is described below, and is performed in 4 steps:

[Step 1] Input of data

Input data are entered into the computer. As shown in Figure 5, the diagnosis

Table 1. Fuzzy rules for evaluating "Condition state of cracking".

\begin{tabular}{|c|c|c|c|}
\hline \multirow[b]{2}{*}{ No. } & \multicolumn{2}{|c|}{ Antecedents } & \multirow{2}{*}{$\begin{array}{c}\text { Consequents } \\
\text { Condition state of cracking } \\
\text { (soundness score) }\end{array}$} \\
\hline & Crack conditions & Maximum crack width & \\
\hline 1 & serious & huge & 0.0 \\
\hline 2 & serious & large & 7.5 \\
\hline 3 & serious & small & 49.9 \\
\hline 4 & serious & OK & 60.1 \\
\hline 5 & not serious & huge & 20.8 \\
\hline 6 & not serious & large & 28.2 \\
\hline 7 & not serious & small & 69.9 \\
\hline 8 & not serious & OK & 76.1 \\
\hline 9 & $\mathrm{OK}$ & huge & 40.8 \\
\hline 10 & $\mathrm{OK}$ & large & 52.8 \\
\hline 11 & $\mathrm{OK}$ & small & 75.6 \\
\hline 12 & OK & OK & 100.0 \\
\hline
\end{tabular}

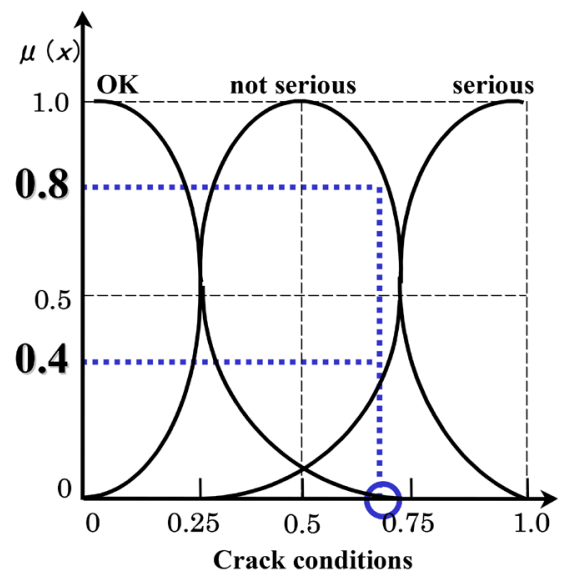

(a)

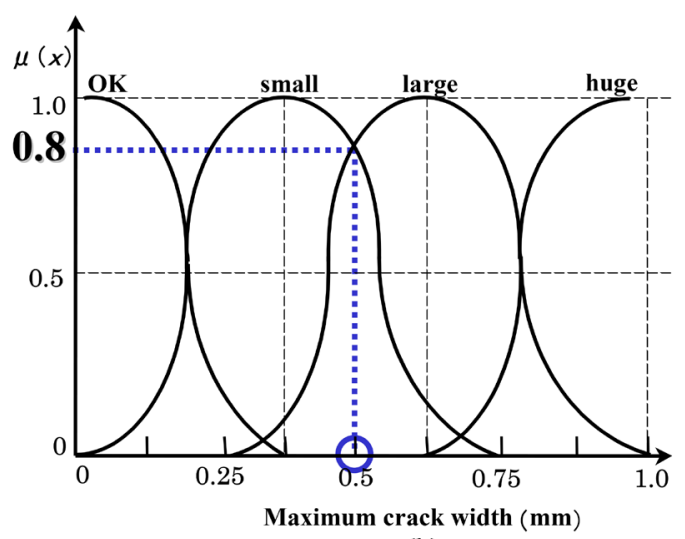

(b)

Figure 6. Fuzzy sets used in antecedents. (a) Membership function for crack conditions; (b) Membership function for maximum crack width. 
of "Condition state of cracking" requires the input data [Crack conditions] and [Maximum crack width $(\mathrm{mm})]$. In the present study, these input data are acquired by simple visual inspection (see Table 2). Therefore, the values of G1-1 and G1-2 in Table 2; that is, 0.7 and $0.5(\mathrm{~mm})$, are used as the input data for the diagnosis.

[Step 2] Calculate the grade of membership functions used in antecedents (see Figure 6 and Figure 7)

The rules of the expert system employ some fuzzy propositions in antecedents of "If-then" rules. In the present study, a fuzzy set is expressed by membership functions. Consequently, from the values of input data for evaluating a judgment item, the grades of membership functions used in antecedents are first calculated. In this example, since the inspection value of [Crack conditions] is 0.7 , this value matches two membership functions, which express the fuzzy set for not serious\} and that for \{serious\}. Therefore, these grades of membership functions are 0.8 and 0.4, respectively (see Figure 6(a)). However, the grade of membership function that expresses the fuzzy set for $\{\mathrm{OK}\}$ is 0.0 , because the inspection value doesn't match the membership function. Similarly, considering the inspection value of [Maximum crack width $(\mathrm{mm})$ ], which is 0.5 , the value also matches two membership functions, which express the fuzzy set for $\{$ small $\}$ and that for \{large\}. Therefore, these grades of membership functions are both 0.8 (see Figure $6(\mathrm{~b}))$. The other grades of membership functions are 0.0 , because the value doesn't match the other fuzzy sets; namely, $\{\mathrm{OK}\}$ and $\{$ huge $\}$. The left-hand section table in Figure 7 indicates the fitness of each fuzzy proposition in antecedents to the inspection results; namely, [Crack conditions] $=0.7$ and [Maximum crack width $(\mathrm{mm})]=0.5$.

[Step 3] Calculate the fitness of each rule to input values (see Figure 7)

Whereas Step 2 calculates the fitness of each fuzzy proposition in antecedents to input values, Step 3 calculates the fitness of each rule to input values. As

Table 2. Partial inspection sheets.

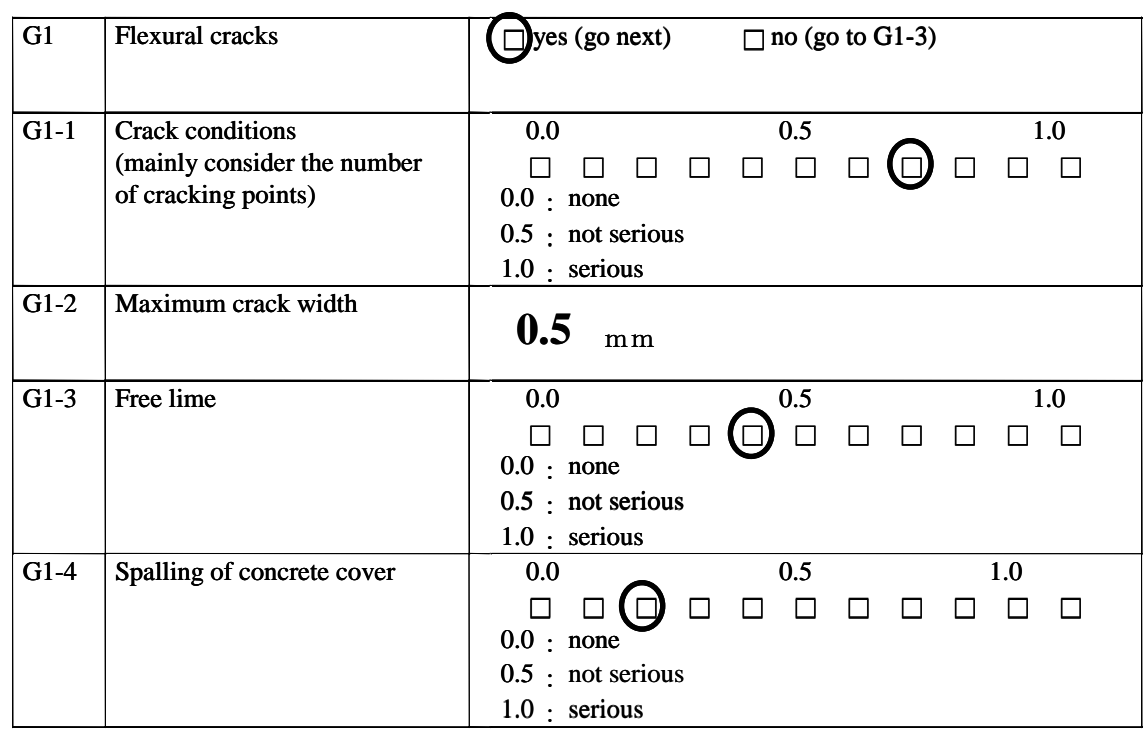




\begin{tabular}{|c|c|c|}
\hline \multirow{2}{*}{ No. } & \multicolumn{2}{|c|}{ Antecedents } \\
\cline { 2 - 3 } & Crack conditions & $\begin{array}{c}\text { Maximum crack } \\
\text { width }\end{array}$ \\
\hline 1 & serious $(0.4)$ & huge $(0.0)$ \\
\hline 2 & serious $(0.4)$ & large $(0.8)$ \\
\hline 3 & serious $(0.4)$ & small $(0.8)$ \\
\hline 4 & serious $(0.4)$ & OK $(0.0)$ \\
\hline 5 & not serious $(0.8)$ & huge $(0.0)$ \\
\hline 6 & not serious $(0.8)$ & large $(0.8)$ \\
\hline 7 & not serious $(0.8)$ & small $(0.8)$ \\
\hline 8 & not serious $(0.8)$ & OK $(0.0)$ \\
\hline 9 & OK $(0.0)$ & huge $(0.0)$ \\
\hline 10 & OK $(0.0)$ & large $(0.8)$ \\
\hline 11 & OK $(0.0)$ & small $(0.8)$ \\
\hline 12 & OK $(0.0)$ & OK $(0.0)$ \\
\hline
\end{tabular}

$(0.4) \times(0.0)=0.0$

$(0.4) \times(0.8)=0.32$

$(0.4) \times(0.8)=0.32$

$(0.4) \times(0.0)=0.0$

$(0.8) \times(0.0)=0.0$

$(0.8) \times(0.8)=0.64$

$(0.8) \times(0.8)=0.64$

$(0.8) \times(0.0)=0.0$

$(0.0) \times(0.0)=0.0$

$(0.0) \times(0.8)=0.0$

$(0.0) \times(0.8)=0.0$

$(0.0) \times(0.0)=0.0$

\begin{tabular}{|c|}
\hline $\begin{array}{c}\text { Fitness of } \\
\text { rule }\end{array}$ \\
\hline 0.0 \\
\hline 0.17 \\
\hline 0.17 \\
\hline 0.0 \\
\hline 0.0 \\
\hline 0.33 \\
\hline 0.33 \\
\hline 0.0 \\
\hline 0.0 \\
\hline 0.0 \\
\hline 0.0 \\
\hline 0.0 \\
\hline
\end{tabular}

[Step 1]\&[Step 2]

[Step 3]

Figure 7. Fuzzy inference process (1).

shown in Figure 7, the fitness of each rule employs the following equations from the grades of membership functions estimated in Step 2:

$$
\begin{gathered}
\hat{\mu}_{i}=\frac{\mu_{i}}{\sum_{k=1}^{n} \mu_{k}} \\
\mu_{i}=\prod_{j} \mu_{i_{j} j}\left(x_{j}\right)
\end{gathered}
$$

where, $\hat{\mu}_{i}$ : fitness of $i$ th rule to input values, such as inspection results.

$\mu_{i_{j} j}\left(x_{j}\right)$ : grade of a membership function.

$i$ : identification number of fuzzy rule.

$j$ : identification number of input variable and fuzzy variable.

$x_{j}$ : input variable.

$\mu_{i_{j} j}$ : fuzzy variable for input variable.

$i_{j}$ : identification number of fuzzy set on fuzzy variable.

$n:$ the number of fuzzy rules.

Equation (18) indicates that all fitness values of fuzzy propositions in the same fuzzy rule are multiplied; that is to say, all grades of membership functions in the same rule are multiplied. Therefore, when the inspection results [Crack conditions] $=0.7$ and [Maximum crack width $(\mathrm{mm})]=0.5$ are entered into the system, the values given in the right-hand section in Figure 7 are estimated by Equation (17) and Equation (18). Rule No. 2 and Rule No. 3 both have a fitness of $17 \%$, and Rule No.6 and Rule No.7 both have a fitness of 33\%.

\section{[Step 4] Calculate a soundness score for a judgment item (Figure 8)}

In the final step, a soundness score for a judgment item is calculated from the fitness of rule acquired in Step 3 and soundness scores described in consequents of fuzzy rules. A soundness score for input values is estimated by the following equation: 


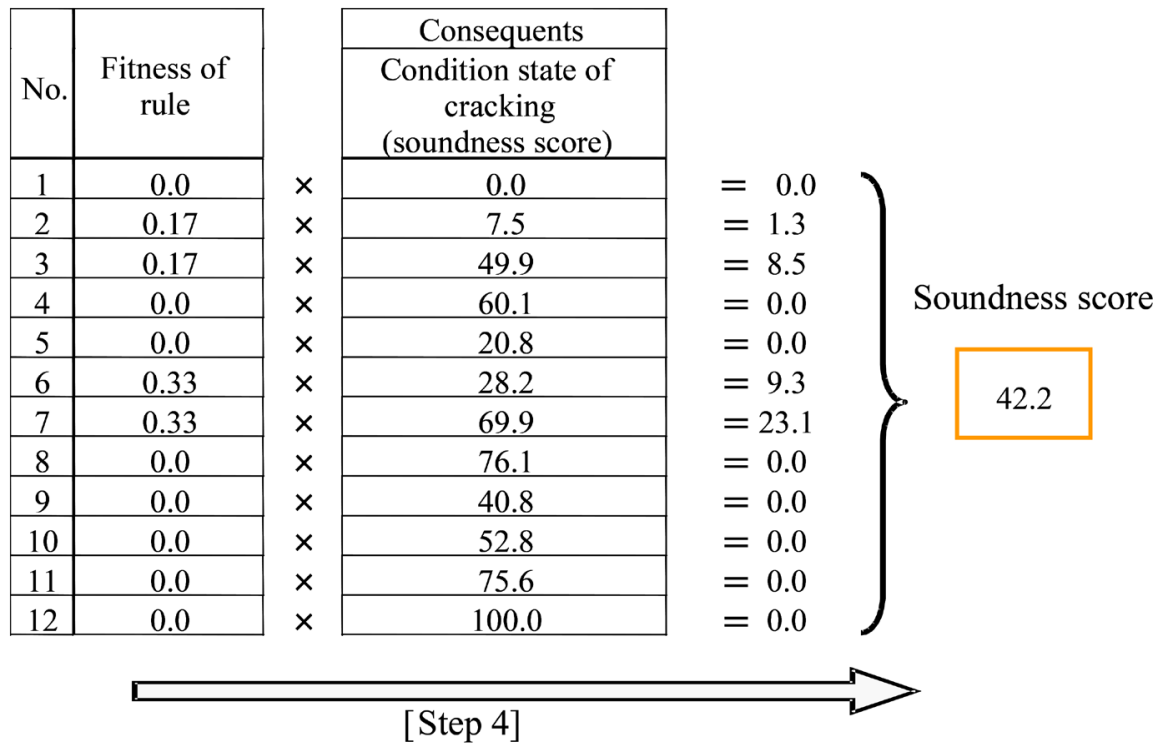

Figure 8. Fuzzy inference process (2).

$$
y=\sum_{k=1}^{n} \hat{\mu}_{k} \omega_{k}
$$

where, $\hat{\mu}_{k}$ : fitness value of $k$ th rule, which is acquired by Equation (17).

$\omega_{k}$ : soundness score described in consequents of $k$ th fuzzy rule.

Consequently, a judgment item is assigned a soundness score on a scale of 0 100. For example, when the input [Crack conditions] $=0.7$ and [Maximum crack width $(\mathrm{mm})]=0.5$ is entered, the expert system outputs the soundness score of 42.2 as the result of diagnosis of input data (see Figure 8).

\subsection{Fuzzy Inference Based on Neural Network}

Structure of fuzzy inference system using a hierarchical neural network. the expert system, the inference mechanism for evaluating a judgment item is constructed with a hierarchical neural network consisting of 5 layers, as shown in Figure 9 [15] [3]. The knowledge for diagnosing "Condition state of cracking"; that is to say, Table 1 and Figure 6 (fuzzy rules and membership functions for fuzzy sets), are implemented in the computer by the neural network shown in Figure 9. Therefore, the neural network can carry out the fuzzy inference mentioned in the previous section. In the present study, the layers of the network are referred to as layers (A), (B), (C), (D) and (E), respectively. These layers have neurons of three different types. The neurons in layers (A), (C) and (E) are linear neurons. The neurons in layer (B) are sigmoid neurons. The neurons in layer (D) are referred to as normalization neurons which employ Equation (17). The Arabic numerals in the layer (D) neurons correspond to the number (No.) in Table 1. Therefore, clearly the connections from layer (C) to layer (E) express a fuzzy rule. A boxed value represents the initial connection weight between neurons or the initial threshold for a neuron.

Next, is described the manner in which the initial values of weight and threshold 


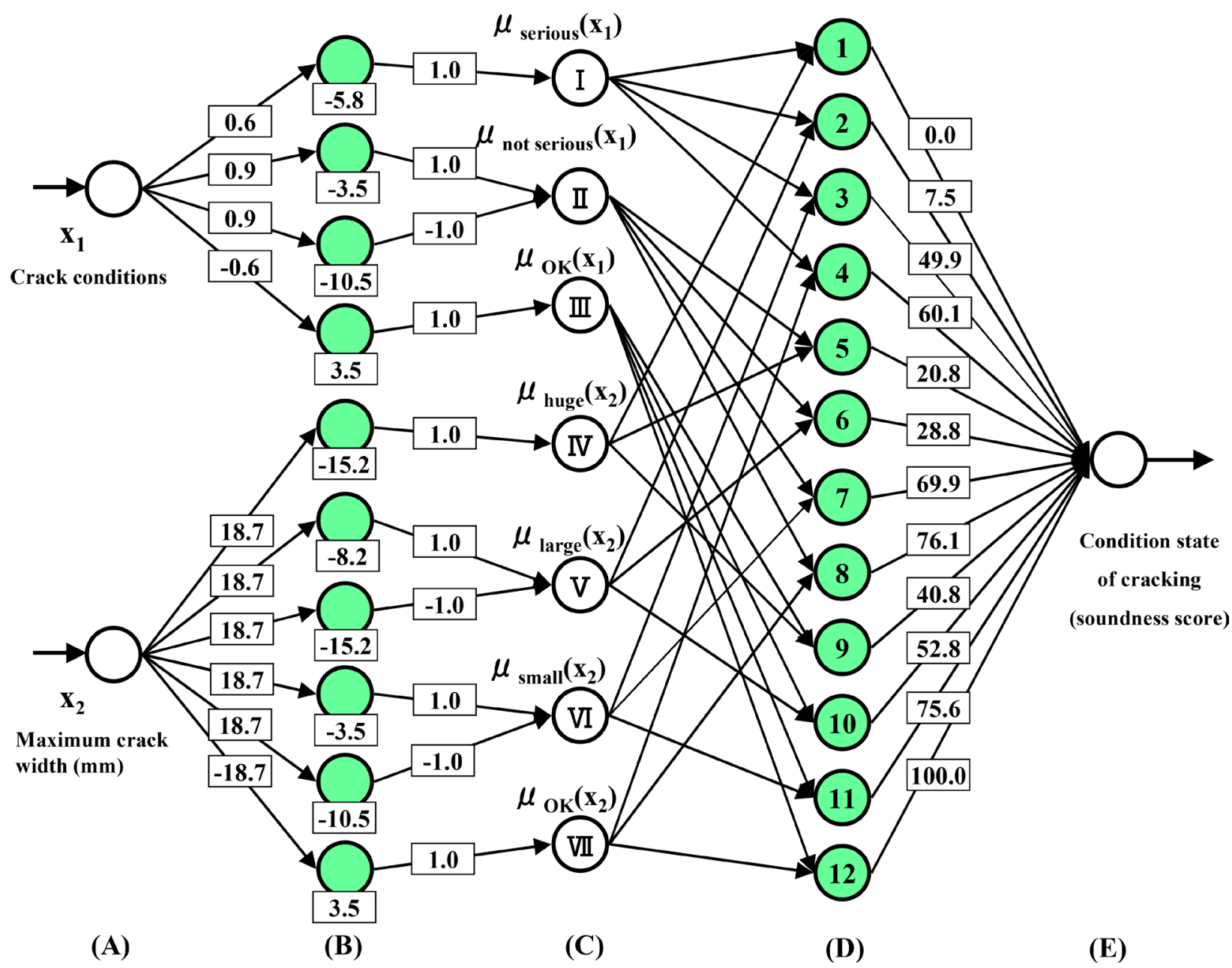

Figure 9. Hierarchical neural network for evaluating "Condition state of cracking”.

are set. The layers (A)-(B)-(C) in the network identify the fuzzy sets in antecedents of fuzzy rules. If the membership function of a fuzzy set is an increasing function or a decreasing function, the form is identified by a sigmoid function; a sigmoid neuron is employed in layer (B) for an increasing function or a decreasing function. If the membership function is a convex function, the form is identified by the combination of two sigmoid functions; two sigmoid neurons are employed in layer (B) for a convex function. Then, the weights $(\omega)$ between layer (A) neurons and layer (B) neurons, and the thresholds $(\theta)$ of the (B) neurons are calculated according to the following equations:

1) Approximation of decreasing function

$$
\left\{\begin{array}{l}
\omega=-h / A \\
\theta=h B / A \\
x_{1}=B-A, \mu\left(x_{1}\right)=1.0, x_{1} \in X \\
x_{2}=B+A, \mu\left(x_{2}\right)=0.0, x_{2} \in X \\
x_{1}, x_{2} \in X
\end{array}\right.
$$

2) Approximation of increasing function 


$$
\left\{\begin{array}{l}
\omega=h / A \\
\theta=-h B / A \\
x_{1}=B+A, \mu\left(x_{1}\right)=1.0, x_{1} \in X \\
x_{2}=B-A, \mu\left(x_{2}\right)=0.0, x_{2} \in X \\
x_{1}, x_{2} \in X
\end{array}\right.
$$

3) Approximation of convex function

$$
\left\{\begin{array}{l}
\omega_{1}=h / A \\
\omega_{2}=h / C \\
\theta_{1}=-h(B-A) / A \\
\theta_{2}=-h(B+C) / C \\
x_{1}=B, \mu\left(x_{1}\right)=1.0 \\
x_{2}=B-2 A, \mu\left(x_{2}\right)=0.0 \\
x_{3}=B+2 C, \mu\left(x_{3}\right)=0.0 \\
x_{1}, x_{2}, x_{3} \in X, x_{2}<x_{3}
\end{array}\right.
$$

Note that $h$ is a real number, which satisfies $f(h) \approx 0$. where, $f(h):$ sigmoid function. In the present study, $h=3.5$. In the case of approximation of an increasing function or a decreasing function, the weights between layer (B) neurons and layer $(\mathrm{C})$ neurons are set to 1.0. In the case of approximation of a convex function, the weights between layer (B) neurons and layer $(\mathrm{C})$ neurons are set to -1.0 for smaller threshold and 1.0 for larger threshold. In addition, initial weights between layer (C) neurons and layer (D) neurons are all 0.5. The initial weights between layer (D) neurons and layer (E) neurons are set according to Table 1. These weights express soundness scores described in consequents of fuzzy rules. Consequently, when input data are entered into the system, layers (A)-(B)-(C) perform the processing of [Step 1] and [Step 2] described earlier. Next, layers (C)-(D) perform the processing of [Step 3]. Finally, layers (D)-(E) perform the processing of [Step 4].

Modification of Fuzzy Rule by Machine Learning. In the hierarchical network shown in Figure 9, each weight and threshold is set for a specific purpose as mentioned above. Therefore, the network is capable of modifying fuzzy rules by altering these parameters, such as weight and threshold. Thus, applying the Back Propagation algorithm to the network as a machine learning method is easy, because the structure of neural network is hierarchical. More specifically, the elements modified by machine learning are the weights between layer (A) neurons and layer (B) neurons, the thresholds of layer (B) neurons, and the weights between layer (D) neurons and a layer (E) neuron. The weights of layers (A)-(B) and the thresholds of layer (B) neurons are used in order to express membership functions in antecedents of fuzzy rules. Consequently, weight alteration after learning indicates the slope alteration of the corresponding membership function, and threshold alteration after learning indicates the axis movement of the membership function in the horizontal direction. In the learning of layers (D)-(E) weight, the proposition in consequents of fuzzy rules is 
changed. For instance, if the weight between a layer (D) neuron and a layer (E) neuron is changed from 0.0 to 1.0 , the proposition described in consequents of fuzzy rule is changed from ([Condition state of cracking] is 0.0 ) to ([Condition state of cracking] is 1.0).

\subsection{Verification of Effectiveness of Machine Learning}

The proposed expert system is developed in Visual Basic and C programming languages and runs on a personal computer. In this section, the expert system is applied to seven existing bridges (nine spans), all of which are RC T-girder-type bridges, in order to test validity of the learning capability. These target bridges stand in Yamaguchi Prefecture, Japan.

Questionnaire survey of domain experts and Visual inspection of bridges: The purpose of the questionnaire survey of domain experts is to acquire teacher data necessary for learning, whereas, the purpose of visual inspection of bridges is to collect inspection data to be entered into the system. The domain experts also use the inspection results to fill out the questionnaires. The results of questionnaire survey and visual inspection were used as training data for carrying out machine learning. In the present study, for collecting training data, visual inspection of bridges and the questionnaire survey were conducted over 2 days. Seven domain experts from four construction consulting companies in and around Yamaguchi Prefecture, Japan participated in the survey. The survey covered nine spans of seven bridges. One set of survey forms, prepared for each span, consists of three different handouts; inspection record sheets (8 pages) to be used to record visual inspection results, a model drawing of each bridge on which the respondents write down whatever comes to mind during inspection, and questionnaire sheets (10 pages) to obtain teacher data required for machine learning. The inspection record sheets are formatted so that the respondents can choose a score from an 11-point rating scale ranging from 0.0 to 1.0 in increments of 0.1 , answer multiple-choice questions, and enter numerical values (Table 2). The questionnaire sheets are formatted so that the respondents can answer in the form of a score on a 0 - 100 scale in increments of 5 points (Figure 10).

Practical application and Verification of the expert system: Table 3 summarizes the questionnaire results of main girder diagnosis by domain experts.

(1) What is the damage degree of flexural cracks?

Please check off the condition after evaluating according to Fig.1.

Damage degree of flexural cracks

\begin{tabular}{|c|c|c|c|c|c|c|c|c|c|c|c|c|c|c|c|c|c|c|c|c|c|c|}
\hline \multicolumn{3}{|c|}{ Danger } & \multicolumn{4}{|c|}{$\underline{\mathrm{S} \text { danger }}$} & \multicolumn{6}{|c|}{ Moderate } & & \multicolumn{5}{|c|}{$\underline{\mathrm{F} \text { safe }}$} & \multicolumn{4}{|c|}{$\underline{\underline{\text { Safe }}}$} \\
\hline 0 & 5 & 10 & 15 & 20 & 25 & 30 & 35 & 40 & 45 & 50 & 5 & & & 65 & 70 & 75 & 80 & 8 & & & 95 & 100 \\
\hline
\end{tabular}

Figure 10. Partial questionnaire sheets. 
The numerical values in parentheses represent averages of scores assigned by the four domain experts, out of the total of seven, who have more than 10 years' experience. The letters S, f-s, M, s-d, and D in the table represent safe, fairly safe, moderate, slightly dangerous, and dangerous. These labels classify the average values in parentheses into five categories, the criteria used by the respondents for this categorization having been mentioned earlier. A number appearing after a bridge name indicates span number. Table 4 and Table 5 present the diagnosis

Table 3. Results of main girder diagnosis by domain experts (Teacher data).

\begin{tabular}{|c|c|c|c|c|c|c|c|c|c|}
\hline $\begin{array}{l}\text { Bridge name } \\
\text { Judgment item }\end{array}$ & HA(1) & NI6) & NO(1) & MI(1) & MI(3) & GE(3) & TO(1) & TO(2) & OU(2) \\
\hline Girder design & $\mathrm{M}(57.5)$ & $\mathrm{M}(46.3)$ & $\mathrm{M}(57.5)$ & $f-s(66.3)$ & $\mathrm{f}-\mathrm{s}(65.0)$ & f-s $(68.8)$ & f-s (72.5) & f-s $(72.5)$ & f-s $(76.3)$ \\
\hline Girder execution & $\mathrm{M}(51.3)$ & s-d (31.3) & f-s $(65.0)$ & f-s (71.3) & f-s $(63.8)$ & f-s (71.3) & f-s (71.3) & $\mathrm{M}(50.0)$ & f-s $(72.5)$ \\
\hline Service conditions & f-s $(67.5)$ & $\mathrm{M}(45.0)$ & f-s (68.8) & f-s (78.8) & f-s $(81.3)$ & f-s $(80.0)$ & f-s (76.3) & f-s $(75.0)$ & f-s $(75.0)$ \\
\hline Material deterioration & $\mathrm{M}(50.0)$ & s-d (35.0) & f-s (78.8) & f-s $(78.8)$ & f-s $(70.0)$ & f-s (86.3) & f-s (81.3) & f-s (68.8) & f-s (82.5) \\
\hline Flexural cracks & f-s (76.3) & s-d (35.0) & f-s $(78.8)$ & f-s (77.5) & f-s (68.8) & f-s $(85.0)$ & f-s (81.3) & f-s (73.8) & f-s $(71.3)$ \\
\hline Shear cracks & $\mathrm{S}(95.0)$ & f-s $(66.3)$ & S (96.3) & $S(97.5)$ & S (93.8) & $S(97.5)$ & $\mathrm{S}(93.8)$ & $S(97.5)$ & $\mathrm{S}(97.5)$ \\
\hline Corrosion cracks & $\mathrm{M}(41.3)$ & $\mathrm{M}(47.5)$ & S (87.5) & f-s $(82.5)$ & f-s (67.5) & $S(90.0)$ & f-s (77.5) & $\mathrm{M}(57.5)$ & f-s $(75.0)$ \\
\hline Bond cracks & $\mathrm{S}(90.0)$ & f-s $(80.0)$ & $S(92.5)$ & $S(87.5)$ & f-s $(85.0)$ & $S(90.0)$ & $\mathrm{S}(92.5)$ & $S(90.0)$ & $\mathrm{S}(90.0)$ \\
\hline Whole damage & $\mathrm{M}(52.5)$ & s-d (26.3) & f-s $(82.5)$ & f-s $(77.5)$ & $\mathrm{f}-\mathrm{s}(70.0)$ & f-s $(86.3)$ & f-s $(76.3)$ & $f-s(66.3)$ & f-s $(77.5)$ \\
\hline Load carrying capability & $f-s(66.3)$ & s-d (36.3) & f-s (71.3) & f-s $(75.0)$ & $\mathrm{f}-\mathrm{s}(71.3)$ & f-s $(81.3)$ & f-s $(72.5)$ & $f-s(66.3)$ & f-s $(80.0)$ \\
\hline Durability & $\mathrm{M}(51.3)$ & s-d (28.8) & f-s $(73.8)$ & f-s $(78.8)$ & f-s $(70.0)$ & f-s $(85.0)$ & f-s $(73.8)$ & f-s $(62.5)$ & f-s $(78.8)$ \\
\hline Serviceability & f-s $(62.5)$ & $\mathrm{s}-\mathrm{d}(30.0)$ & f-s $(71.3)$ & $\mathrm{f}-\mathrm{s}(75.0)$ & f-s $(65.0)$ & f-s $(85.0)$ & f-s (72.5) & f-s $(66.3)$ & f-s $(80.0)$ \\
\hline
\end{tabular}

Note; S: safe (87.6 - 100.0), f-s: fairly-safe (62.6 - 87.5), M: moderate (37.6 - 62.5), s-d: slightly-dangerous (12.6 - 37.5$)$, D: dangerous (0 - 12.5).

Table 4. Results of main girder diagnosis by the initial system before learning.

\begin{tabular}{|c|c|c|c|c|c|c|c|c|c|}
\hline $\begin{array}{l}\text { Bridge name } \\
\text { Judgment item }\end{array}$ & HA(1) & $\mathrm{NI}(6)$ & NO(1) & MI(1) & MI(3) & GE(3) & TO1) & TO(2) & OU(2) \\
\hline Girder design & $\mathrm{M}(60.3)$ & M (58.5) & M (50.5) & M (59.9) & M (59.9) & $\mathrm{M}(62.0)$ & $\mathrm{M}(56.1)$ & $\mathrm{M}(56.1)$ & $\mathrm{M}(54.1)$ \\
\hline Girder execution & $\mathrm{M}(44.9)$ & M (41.4) & f-s $(69.6)$ & $f-s(69.6)$ & $\mathrm{M}(51.8)$ & M (46.6) & $\mathrm{M}(51.8)$ & $\mathrm{M}(51.8)$ & $\mathrm{M}(51.8)$ \\
\hline Service conditions & $S(92.9)$ & $S(92.7)$ & S (91.4) & S (97.4) & S (97.4) & S (94.9) & S (97.5) & S (97.5) & S (97.4) \\
\hline Material deterioration & s-d (32.0) & $s-d(25.1)$ & $\mathrm{M}(51.0)$ & f-s $(66.3)$ & M (59.2) & $\mathrm{M}(47.1)$ & M (52.9) & s-d (28.9) & M (48.7) \\
\hline Flexural cracks & $\mathrm{M}(51.2)$ & s-d (16.7) & M (53.9) & $S(96.1)$ & $\mathrm{S}(91.8)$ & $S(87.5)$ & S (91.1) & f-s $(67.5)$ & $S(95.2)$ \\
\hline Shear cracks & S (99.9) & S (99.9) & S (99.9) & S (99.9) & S (99.9) & S (99.9) & S (99.9) & S (99.9) & S (99.9) \\
\hline Corrosion cracks & s-d (20.3) & $\mathrm{D}(9.1)$ & S (99.9) & S (99.9) & $\mathrm{M}(52.3)$ & S (99.9) & S (99.9) & $\mathrm{D}(7.1)$ & M (57.8) \\
\hline Bond cracks & S (99.9) & S (99.9) & S (99.9) & S (99.9) & S (99.9) & S (99.9) & $S(99.9)$ & S (99.9) & $S(99.9)$ \\
\hline Whole damage & s-d (23.3) & s-d (27.7) & f-s $(67.4)$ & f-s $(80.2)$ & $M(55.1)$ & $M(58.2)$ & f-s $(65.4)$ & $\mathrm{s}-\mathrm{d}(15.1)$ & M (47.4) \\
\hline Load carrying capability & $\mathrm{f}-\mathrm{s}(70.8)$ & f-s $(74.1)$ & $S(89.0)$ & S (89.4) & S (89.0) & f-s (74.9) & f-s $(67.3)$ & M (47.6) & f-s $(73.1)$ \\
\hline Durability & $\mathrm{s}-\mathrm{d}(22.1)$ & s-d (29.7) & f-s $(62.7)$ & f-s (83.7) & $M(52.2)$ & $M(53.9)$ & $M(60.1)$ & $\mathrm{D}(10.0)$ & $\mathrm{M}(48.9)$ \\
\hline Serviceability & M (43.9) & M (53.3) & f-s (75.6) & S (91.4) & f-s $(72.4)$ & f-s $(63.2)$ & M (58.9) & s-d (27.2) & $M(60.6)$ \\
\hline Total error & 195.0 & 253.5 & 158.5 & 128.4 & 161.0 & 197.7 & 174.6 & 311.1 & 238.8 \\
\hline
\end{tabular}


Table 5. Results of main girder diagnosis by the system after learning.

\begin{tabular}{|c|c|c|c|c|c|c|c|c|c|}
\hline $\begin{array}{l}\text { Bridge name } \\
\text { Judgment item }\end{array}$ & HA(1) & $\mathrm{NI}(6)$ & $\mathrm{NO}(1)$ & MI(1) & $\mathrm{MI}(3)$ & GE(3) & TO 1 & $\mathrm{TO}(2)$ & OU(2) \\
\hline Girder design & f-s $(65.4)$ & f-s $(66.7)$ & f-s $(67.6)$ & f-s $(65.1)$ & f-s $(65.1)$ & f-s (64.5) & f-s (66.6) & f-s $(66.6)$ & f-s $(67.7)$ \\
\hline Girder execution & M (53.0) & M (39.3) & f-s $(69.4)$ & f-s $(69.4)$ & f-s $(66.6)$ & $\mathrm{M}(56.5)$ & f-s $(66.6)$ & f-s $(66.6)$ & f-s (66.6) \\
\hline Service conditions & M (59.4) & M (59.5) & f-s $(68.0)$ & f-s $(80.4)$ & f-s $(80.4)$ & f-s $(71.4)$ & f-s $(76.3)$ & f-s $(76.3)$ & f-s (77.5) \\
\hline Material deterioration & M (58.7) & $\mathrm{M}(40.1)$ & f-s $(79.2)$ & f-s $(78.7)$ & f-s $(72.5)$ & f-s (76.8) & f-s $(80.0)$ & $\mathrm{M}(61.2)$ & f-s (84.6) \\
\hline Flexural cracks & f-s $(75.0)$ & s-d (35.0) & f-s $(70.7)$ & f-s $(76.6)$ & f-s $(77.2)$ & f-s (77.8) & f-s $(76.1)$ & f-s $(74.0)$ & f-s $(77.0)$ \\
\hline Shear cracks & S (93.3) & S (93.3) & $S(93.3)$ & S (93.3) & S (93.3) & $S(93.3)$ & S (93.3) & S (93.3) & $S(93.3)$ \\
\hline Corrosion cracks & M (43.8) & M (51.6) & f-s $(84.9)$ & f-s $(84.9)$ & f-s $(66.8)$ & f-s (84.9) & f-s $(84.9)$ & M (52.5) & f-s (75.5) \\
\hline Bond cracks & S (90.1) & S (90.1) & S (90.1) & S (90.1) & $\mathrm{S}(90.1)$ & $S(90.1)$ & S (90.1) & $\mathrm{S}(90.1)$ & $S(90.1)$ \\
\hline Whole damage & M (57.6) & s-d (27.8) & f-s $(79.0)$ & f-s $(79.2)$ & f-s $(71.7)$ & f-s $(78.4)$ & f-s $(79.6)$ & $\mathrm{M}(60.9)$ & f-s (78.7) \\
\hline Load carrying capability & f-s $(75.7)$ & M (53.3) & f-s $(79.4)$ & f-s $(77.7)$ & f-s (72.9) & f-s $(68.1)$ & f-s (67.9) & M (54.4) & f-s (71.3) \\
\hline Durability & $\mathrm{M}(52.0)$ & s-d (31.7) & f-s $(75.9)$ & f-s $(79.8)$ & f-s $(70.5)$ & f-s $(76.1)$ & f-s $(79.3)$ & $\mathrm{M}(58.0)$ & $\mathrm{f}-\mathrm{s}(78.5)$ \\
\hline Serviceability & f-s $(67.6)$ & M (40.3) & f-s $(77.7)$ & f-s $(78.4)$ & f-s $(70.6)$ & f-s $(69.2)$ & f-s (69.9) & M (58.1) & f-s $(72.3)$ \\
\hline Total error & 52.3 & 120.9 & 51.9 & 23.7 & 30.4 & 99.6 & 43.4 & 70.9 & 47.5 \\
\hline
\end{tabular}

results of main girders before learning and after learning, respectively. As mentioned earlier, in the present study, the Back Propagation method was applied as a learning method. The re-substitution method was applied to the system as a training method. The training method uses all combinations of questionnaire survey and visual inspection; nine sets for nine spans, as training data for machine learning. Therefore, the data of a diagnosed bridge also include the training data. Evaluating the judgment items of a target bridge span on the basis of knowledge modified by the above training method is equivalent to evaluating the judgment items of an already-encountered span after completing learning sessions for a number of spans. The shaded areas in the tables indicate the following: gray shading indicates a system output value that deviates one order from the teacher value (see Table 3), and black shading indicates an output value that deviates two or more orders from the teacher value. The total error at the bottom of the table is a span-by-span sum of errors for each judgment item. Comparison of these outputs (Table 4 and Table 5) with the questionnaire survey results (Table 3 ) reveals that of the 108 judgment items $(9$ spans $\times 12$ judgement items) for the main girders, 42 items before learning and 88 items after learning show agreement with the questionnaire results, 58 items before learning and 20 items after learning show deviation of one order from the teacher value, and 8 items before learning show deviation of two or more orders from the teacher value. Thus, the total agreement ratios before learning and after learning are 38.9 and 81.5 percent, respectively. Improvement of agreement ratio shows the validity of applying the machine learning method to the system. However, since the reliability of the system depends on information on the distribution of bridge damage used for neural network learning, we must increase the number of sam- 
ple bridge data sets used for learning and acquire data sets for various damage conditions.

Next, modification of fuzzy rules is shown in order to verify the effectiveness of the applied neural network structure. Refinement of fuzzy rules for evaluating "Condition state of cracking" is presented as an example. Figure 11 and Figure 12 show the membership functions used in antecedents of fuzzy rules before learning and after learning, respectively. The symbols in the figures indicate the following: (1), (2) and (3) indicate the membership functions of fuzzy sets $\{\mathrm{OK}\},\{$ not serious\}, and \{serious\} for input data [Crack conditions], respectively, and I, II, III, and IV indicate the membership functions of $\{\mathrm{OK}\},\{$ small $\},\{$ large $\}$, and \{huge\} for input data [Maximum crack width $(\mathrm{mm})$ ], respectively. Table 6 shows the weight modification between layer (D)-(E) neurons. No. in the table indicates rule number, which corresponds to Table 1. Comparing Figure 11 and Figure 12, we notice that after learning, the horizontal width of membership function I is reduced by $2 / 3$. The reduction indicates that the system after learning treats the input value of [Maximum crack width $(\mathrm{mm})$ ], which is smaller than that before learning, as the fuzzy set of $\{\mathrm{OK}\}$. The other membership functions after

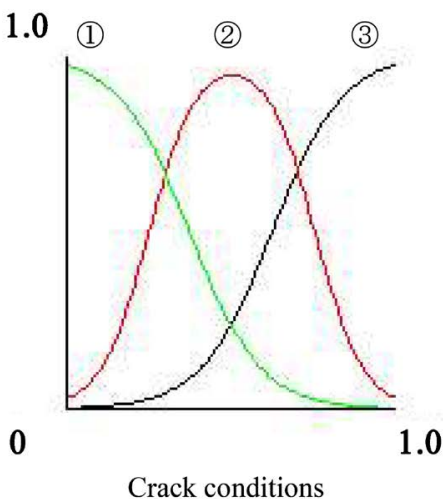

(a)

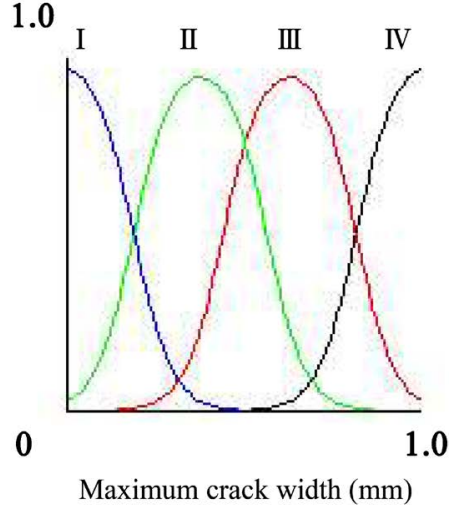

(b)

Figure 11. Fuzzy sets used in antecedents before learning. (a) Membership function for crack condition; (b) Membership function for maximum crack width.

1.0

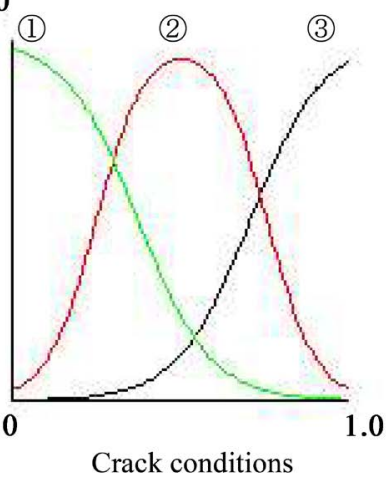

(a)

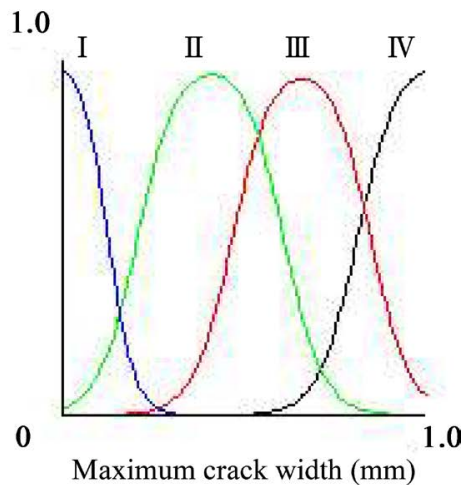

(b)

Figure 12. Fuzzy sets used in antecedents after learning. (a) Membership function for crack condition; (b) Membership function for maximum crack width. 
learning are similar to those before learning. As a result of comparison of the weights before learning and the weights after learning in Table 6, the transition is summarized as follows: The weights of fuzzy rules 1, 2 and 3 after learning are similar. Therefore, the difference between fuzzy sets $\{$ huge $\},\{$ large $\}$, and $\{$ small $\}$ for the input data [Maximum crack width $(\mathrm{mm})$ ] isn't distinguished when the input value of [Crack conditions] entered in the system is categorized into the fuzzy set \{serious\} (see Table 1). The weights of fuzzy rules 5, 6 and 7 after learning are similar. Therefore, the difference between fuzzy sets \{huge\}, \{large\}, and \{small $\}$ for the input data [Maximum crack width $(\mathrm{mm})]$ isn't distinguished when the input value of [Crack conditions] entered in the system is categorized into the fuzzy set \{not serious\}. The weights of fuzzy rules 9,10 and 11 after learning are similar. Therefore, the difference between fuzzy set \{huge\}, \{large\}, and \{small\} for the input data [Maximum crack width $(\mathrm{mm})$ ] clearly isn't distinguished when the input value of [Crack conditions] entered in the system is categorized into the fuzzy set $\{\mathrm{OK}\}$. Consequently, after learning, although the membership functions of fuzzy sets $\{$ small $\}$, \{large $\}$ and $\{$ huge $\}$ for input data [Maximum crack width $(\mathrm{mm})$ ] didn't change, as shown in Figure 11(b) and Figure 12(b). Table 6 indicates that these three categories; fuzzy sets \{small\}, $\{$ large $\}$, and \{huge\}, can be regarded as one category. In other words, the domain experts don't use the four categories; fuzzy sets $\{\mathrm{OK}\},\{$ small $\},\{$ large $\}$ and $\{$ huge $\}$ for input data [Maximum crack width $(\mathrm{mm})$ ] in diagnosing the judgment item "Condition state of cracking"; rather, they use two categories; fuzzy set $\{\mathrm{OK}\}$ and a category that includes $\{$ small\}, \{large\}, and \{huge\}. The weights of fuzzy rules 8 and 12 are similar; therefore the difference between fuzzy sets \{not serious\} and $\{\mathrm{OK}\}$ for input data [Crack conditions] obviously isn't distinguished when the

Table 6. Weight modification between layer (D)-(E) neurons. (Modification of soundness score in consequents of fuzzy rules).

\begin{tabular}{ccc}
\hline \multirow{2}{*}{ No. } & Weights between layer (D)-(E) neurons \\
\cline { 2 - 3 } 1 & Before learning & After learning \\
\hline 2 & 0.0 & 0.0 \\
3 & 7.5 & 0.0 \\
4 & 49.9 & 6.6 \\
5 & 60.1 & 60.1 \\
6 & 20.8 & 20.8 \\
7 & 28.2 & 20.8 \\
8 & 69.9 & 21.0 \\
9 & 76.1 & 76.1 \\
10 & 40.8 & 40.8 \\
11 & 52.8 & 40.8 \\
12 & 75.6 & 42.1 \\
\hline
\end{tabular}


input value of [Maximum crack width (mm)] is categorized into the fuzzy set $\{\mathrm{OK}\}$. These results show that the number of fuzzy rules for evaluating the judgment item "Condition state of cracking" could be reduced by machine learning.

\section{Discussion and Summary}

Knowledge-based systems for the assessment and management conjunction with the latest information technologies will be becoming more challenging issues in the future for maintenance and rehabilitation of existing bridges. In this paper, although several methods are available for it, two modern bridge management systems which include knowledge-based expert system based on data such as inspection results and questionnaire surveys of domain experts incorporation with not only reliability approach but also a hierarchical neural network, fuzzy reasoning, etc. were introduced and comparative reviwed.

In the first part of this paper development of advanced bridge management systems is discussed, with special emphasis on reinforced concrete bridges. Management systems for prestressed concrete bridges, or composite bridges can be developed in a similar way. The proposed procedures are illustrated by the EU-supported management systems BRIDGE1 and BRIDGE2.

In the second part, a concrete bridge rating expert system with machine learning is presented. By application of a hierarchical neural network, the developed system not only performs fuzzy inference but also facilitates refinement of the knowledge base, based on data such as inspection results and questionnaire surveys of domain experts. Moreover, the proposed neural network contributes to preventing the inference mechanism from becoming a black box.

The results of the present study can be summarized as follows:

1) The first knowledge-based system is divided into two modules BRIDGE1 and BRIDGE2 which are used in two different situations, namely by the inspector of the bridge during the inspection at the site and after the inspector has returned to his office. In the bridge management systems BRIDGE1 and $B R I D G E 2$, the updating of stochastic variables etc. is performed using the techniques described earlier.

2) The expert system module BRIDGE1 is used at the bridge site during an inspection. This expert system module contains useful information concerning the bridge inspected and the defects observed. The information includes: general information about the bridge, appropriate diagnostic methods for each defect, probable causes for each defect, and other defects related to a defect. It is also possible to create a provisional defect report.

3) The expert system module BRIDGE2 is used to make a detailed analysis of the bridge after an inspection when testing has been performed in the laboratory. New bridges and cross-sections can be entered into the database and existing bridges and cross-sections can be edited. For the bridges in the database the following options are available: review provisional defect reports, enter inspection 
results, estimate the reliability index, plan maintenance work and estimate costs, plan structural repair work and estimate costs, and review the agenda of inspection for one bridge or all bridges.

4) In the second knowledge-based system, as a method of knowledge base refinement, a learning method based on the hierarchical neural network has been presented. The method prevented the neural network from becoming a black box.

5) J-BMS BREX was applied to the main girders of existing bridges in order to verify the effectiveness of the machine learning method. The knowledge base was refined from the results of questionnaire surveys of domain experts. Close agreement between the teaching values and the values after learning, and favorable results achieved as a result of knowledge base refinement confirm the effectiveness of the learning method in the system. However, in order to enhance the reliability of the expert system, the knowledge base must be refined through application to a greater number of bridges.

The author would hope that this paper would be some help on this field in the future.

\section{Acknowledgements}

The author would like to thank Prof. P.Thoft-Christensen, Professor Emeritus of Aalborg University, Denmark for his great help and advice.

\section{Conflicts of Interest}

The author declares no conflicts of interest regarding the publication of this paper.

\section{References}

[1] Yanev, B. (2007) Bridge Management. John Wiley \& Sons, Inc., New Jersey. https://doi.org/10.1002/9780470168059

[2] Forsyth, R. (1984) Expert Systems-Principles and Case Studies. 2nd Edition, Chapman and Hall Computing, London.

[3] Okada, H., Watanabe, N., Kawamura, A. and Asakawa, K. (1992) Initializing Multi-layer Neural Networks with Fuzzy Logic. [Proceedings 1992] IJCNN International Joint Conference on Neural Networks, Baltimore, 7-11 June 1992, 239-244.

[4] Rumelhart, D.E., Hinton, G.E. and Williams, R.J. (1986) Learning Representations by Back-Propagating Errors. Nature, 323, 533-536. https://doi.org/10.1038/323533a0

[5] Tazaki, E. and Amagasa, M. (1979) Structural Modeling in a Class of Systems Using Fuzzy Sets Theory. International Journal for Fuzzy Sets and Systems, 2, 1-17. https://doi.org/10.1016/0165-0114(79)90018-6

[6] Ang, A.H.S. and Tang, W.H. (1975) Probability Concepts in Engineering Planning and Design, Vol. 1-Basic Principles. John Wily \& Sons, New York.

[7] Thoft-Christensen, P. (1995) Advanced Bridge Management Systems. Structural Engineering Review, 7, 151-163.

[8] Thoft-Christensen, P., Jensen, F.M., Middleton, C.M. and Blackmore, A. (1996) As- 
sessment of the Reliability of Concrete Slab Bridges. Department of Building Technology and Structural Engineering, Aalborg.

[9] Thoft-Christensen, P. (1996) Re-Assessment of Concrete Bridges. Department of Building Technology and Structural Engineering, Aalborg, 613-620.

[10] Miyamoto, A. and Motoshita, M. (2015) Development and Practical Application of a Bridge Management System (J-BMS) in Japan. Civil Engineering Infrastructures Journal (CEI) , 48, 189-216.

[11] Kusida, M., Miyamoto, A. and Kinoshita, K. (1997) Development of Concrete Bridge Rating Prototype Expert System with Machine Learning. ASCE Journal of Computing in Civil Engineering, 11, 238-247. https://doi.org/10.1061/(ASCE)0887-3801(1997)11:4(238)

[12] Miyamoto, A., Morikawa, H., Kusida, M. and Tokuyama, T. (1993) A knowledge-based Expert System Application in Structural Safety Assessment of Concrete Bridges. Bridge Management, 2, 96-109.

[13] Miyamoto, A., Kusida, M. and Kinoshita, K. (1995) Concrete Bridge Rating Expert System with Machine Learning. Processing of IABSE Colloquium on Knowledge Support Systems in Civil Engineering, Bergamo, 16-19 March 1995, 301-306.

[14] Miyamoto, A. and Kusida, M. (1996) Development of Neuro-Fuzzy Expert System for Serviceability Assessment of Concrete Bridges. Technology Reports of Yamaguchi University, 5, 335-353.

[15] Miyamoto, A., Kawamura, K., Nakamura, H. and Yamamoto, H. (2000) Development of Concrete Bridge Rating Expert System by Using Hierarchical Neural Networks. Japan Society of Civil Engineers (JSCE), 644, 67-86. (In Japanese) https://doi.org/10.2208/jscej.2000.644 67

[16] Madsen, H.O. (1987) Model Updating in Reliability Theory. Proceedings of $5^{\text {th }}$ International Conference on Applications of Statistics and Probability in Soil and Structural Engineering (ICASP5), Mexico, 17-21 June 1987, 564-577.

[17] Rackwitz, R. and Schrupp, K. (1985) Quality Control, Proof Testing and Structural Reliability. Structural Safety, 2, 239-244. https://doi.org/10.1016/0167-4730(85)90030-X

[18] Thoft-Christensen, P. and Baker, M.J. (1982) Structural Reliability Theory and Its Applications. Springer Verlag, Berlin. https://doi.org/10.1007/978-3-642-68697-9

[19] Thoft-Christensen, P. and Murotsu, Y. (1986) Application of Structural Systems Reliability Theory. Springer Verlag, Berlin. https://doi.org/10.1007/978-3-642-82764-8

[20] Middleton, C. (1994) Example Collapse \& Reliability Analyses of Concrete Bridges Using a New Analyses Technique. Cambridge University, Cambridge.

[21] RELIAB01, Version 2.0 (1994) Manual and Software, CSR-Software.

[22] RELIAB02, Version 2.0 (1994) Manual and Software, CSR-Software.

[23] CORROSION, Version 2.0 (1996) Manual and Software, CSR-Software.

[24] Morikawa, H., Miyamoto, A. and Takeuchi, K. (1996) Structural Safety Evaluation and Remaining Life Prediction of Concrete Bridges Based on Statistical Analysis. Concrete Library of JSCE, 28, 51-64.

[25] Nishimura, A., Fujii, M. and Miyamoto, A. (1983) Diagnosis of Reinforced Concrete Slabs for Highway Bridges. Memoirs of the Faculty of Engineering Kobe University, 30, 51-69. 